\title{
Synthesis and Activity against Mycobacterium tuberculosis of Olivacine and Oxygenated Derivatives
}

\author{
Ulrike Schmidt ${ }^{1}$, Gabriele Theumer ${ }^{1}$, Anne Jäger ${ }^{1}$, Olga Kataeva ${ }^{2}$, Baojie Wan ${ }^{3}$, \\ Scott G. Franzblau ${ }^{3}$ and Hans-Joachim Knölker ${ }^{1, *}$ (i) \\ 1 Faculty of Chemistry, Technische Universität Dresden, Bergstraße 66, 01069 Dresden, Germany; \\ ulrike.schmidt@chemie.tu-dresden.de (U.S.); Gabriele.Theumer@tu-dresden.de (G.T.); \\ anne.jaeger@chemie.tu-dresden.de (A.J.) \\ 2 A. M. Butlerov Chemistry Institute, Kazan Federal University, Kremlevskaya Str. 18, Kazan 420008, Russia; \\ olga-kataeva@yandex.ru \\ 3 Institute for Tuberculosis Research, College of Pharmacy, University of Illinois at Chicago, \\ 833 S. Wood St., MC 964, Chicago, IL 60612-7231, USA; baojie@uic.edu (B.W.); sgf@uic.edu (S.G.F.) \\ * Correspondence: hans-joachim.knoelker@tu-dresden.de; Fax +49-351-463-37030
}

Received: 30 April 2018; Accepted: 6 June 2018; Published: 9 June 2018

\begin{abstract}
The tetracyclic pyrido[4,3-b]carbazole olivacine and four of its oxygenated derivatives have been synthesized by a late-stage palladium-catalyzed Heck-type cyclization of the pyrrole ring as a key step. In a test for the inhibition of the growth of Mycobacterium tuberculosis, 9-methoxyolivacine showed the most significant inhibitory activity against Mycobacterium tuberculosis, with an $\mathrm{MIC}_{90}$ value of $1.5 \mu \mathrm{M}$.
\end{abstract}

Keywords: inhibitory activity; catalysis; cyclization; olivacine; palladium; pyrido[4,3-b]carbazoles

\section{Introduction}

The pyrido[4,3-b]carbazole alkaloid olivacine (1, Figure 1) was first isolated in 1958 by Schmutz et al. [1], and its structural assignment was confirmed by total synthesis only two years later [2]. The tetracyclic alkaloid 1 and many structurally related compounds, for example the isomeric natural product ellipticine (2), show useful biological activities, such as anti-tumor activity, based on DNA intercalation, topoisomerase II inhibition, and antimalarial activity [3-7]. Since the 1980s, A-ring oxygenated derivatives of ellipticine (2) have attracted much attention because of their anti-tumor activity [8]. Elliptinium acetate (3) has reached the status of a licensed drug for the treatment of advanced breast cancer [9]. Diverse total syntheses of olivacine (1) have been reported [10-18]. Surprisingly, the pharmacological potential of olivacine (1) and its oxygenated derivatives (for example 4 and 5 ) has been much less investigated [19].

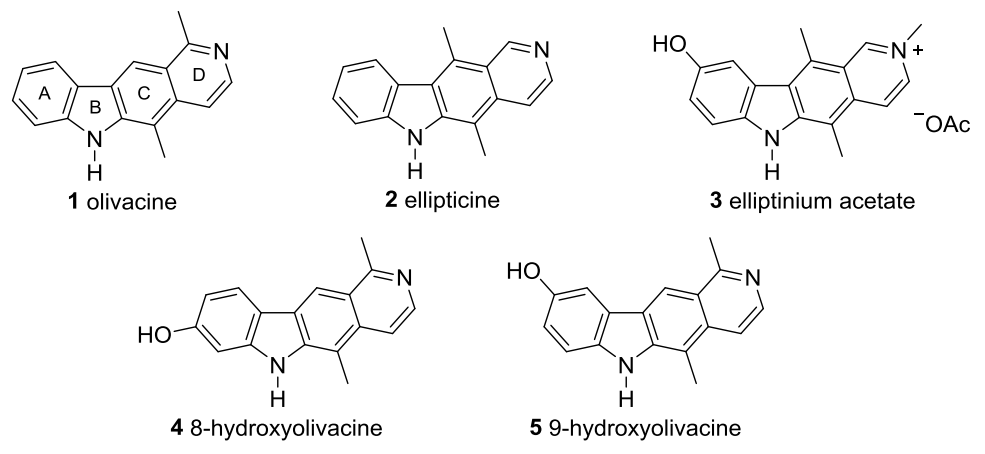

Figure 1. Pyrido[4,3- $b$ ]carbazole alkaloids and oxygenated derivatives. 
Although 9-hydroxyolivacine (5) is the main derivative produced by the metabolic conversion of olivacine (1) [3], derivatives of olivacine (1) with A-ring substitution have been not described extensively in the literature $[3,11,13,20]$. This may be because the syntheses of pyrido[4,3- $b]$ carbazoles usually involve the annulation of an isoquinoline or a pyridine at an indole or carbazole framework $[8,10,11]$. Thus, a facile variation of the substitution pattern at ring $\mathrm{A}$ is not easy to accomplish. Herein, we present a novel route for the synthesis of the tetracyclic pyrido[4,3-b]carbazole framework [21].

\section{Results and Discussion}

For a convergent access to various A-ring substituted derivatives, we envisaged a late-stage B-ring construction of the pyrido[4,3-b]carbazole framework. Therefore, we applied the two-step sequence of palladium-catalyzed reactions developed by our group for carbazole assembly: synthesis of a diarylamine via Buchwald-Hartwig coupling of appropriate anilines 7 with a substituted isoquinoline 8 followed by oxidative cyclization to the pyrido[4,3- $b$ ]carbazoles 6 (Scheme 1) [11]. The isoquinoline 8 would be available by Bischler-Napieralski cyclization of the arylethylamine 9 via the corresponding acetamide. Henry reaction of an appropriately substituted benzaldehyde $\mathbf{1 0}$ and subsequent reduction should afford the arylethylamine 9. As the Bischler-Napieralski reaction works best on electron rich aromatic systems, we decided to start from the commercially available methoxy-substituted benzaldehyde $\mathbf{1 1}$ (Scheme 2) and to transform the methoxy group into a suitable leaving group at a later stage of our synthesis.

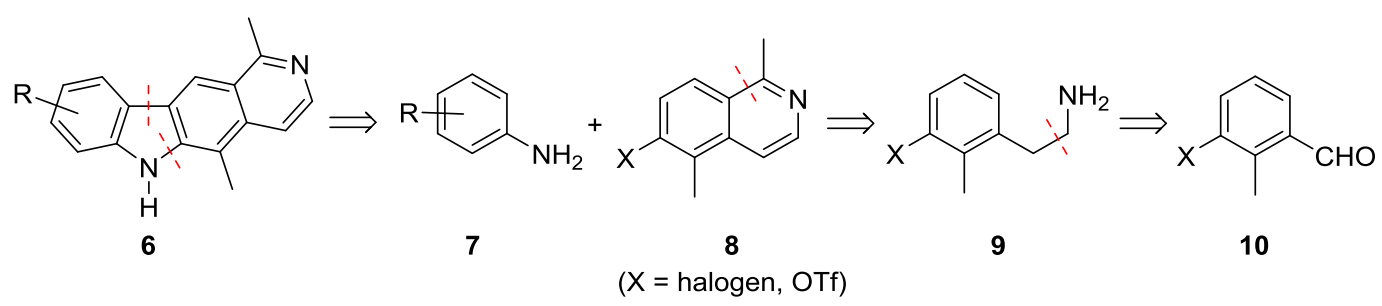

Scheme 1. Retrosynthetic analysis for the pyrido[4,3-b]carbazole olivacine and its A-ring derivatives.

\subsection{Total Synthesis}

Starting from the commercial benzaldehyde 11, which can also be obtained in one step and $87 \%$ yield from the much cheaper $m$-anisaldehyde [22], amide 12 is prepared by a three-step sequence of Henry reaction, lithium aluminum hydride reduction, and N-acetylation (Scheme 2) [23]. Bischler-Napieralski cyclization using phosphorus oxychloride led to the corresponding dihydroisoquinoline, which was fully aromatized to 6-methoxy-1,5-dimethylisoquinoline (13) by dehydrogenation with palladium on charcoal in the presence of cyclohexene as additive. Cleavage of the methyl ether afforded the isoquinolinol, which on reaction with trifluoromethanesulfonic anhydride provided the known isoquinolinyl triflate 14 [24] in $58 \%$ yield over seven steps.

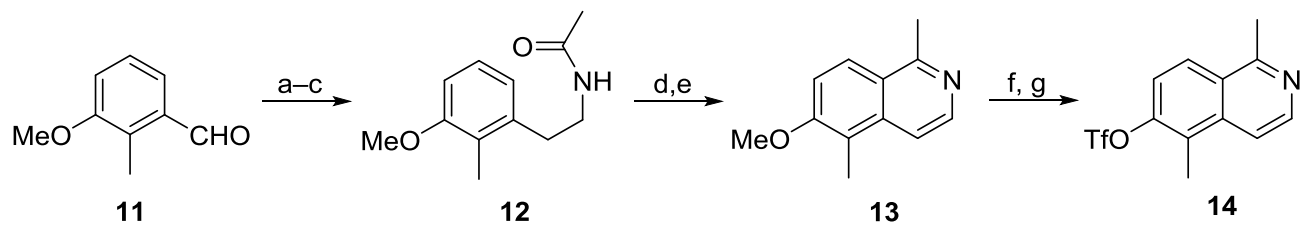

Scheme 2. Synthesis of the triflate 14. Reagents and conditions: (a) $\mathrm{MeNO}_{2}, \mathrm{NH}_{4} \mathrm{OAc}, \mathrm{AcOH}, 80{ }^{\circ} \mathrm{C}$, $110 \mathrm{~min}, 77 \%$; (b) $\mathrm{LiAlH}_{4}, \mathrm{THF}, 0{ }^{\circ} \mathrm{C}$ to reflux, $19.5 \mathrm{~h}, 92 \%$; (c) $\mathrm{Ac}_{2} \mathrm{O}$, cat. DMAP, pyridine, $0{ }^{\circ} \mathrm{C}, 4 \mathrm{~h}$, 99\%; (d) $\mathrm{POCl}_{3}$, reflux, $1 \mathrm{~h}$, 99\%; (e) Pd/C (10\%), cyclohexene, PhMe, reflux, $1.5 \mathrm{~h}, 100 \%$; (f) pyridinium chloride, microwave $(300 \mathrm{~W}), 155^{\circ} \mathrm{C}, 30 \mathrm{~min}, 96 \%$; $(\mathrm{g}) \mathrm{Tf}_{2} \mathrm{O}$, pyridine, $\mathrm{MeCN}, 0{ }^{\circ} \mathrm{C}, 20 \mathrm{~h}, 87 \%$. 
Buchwald-Hartwig coupling [25] of the triflate 14 and aniline (15) provided the diarylamine 16 (Scheme 3). However, the oxidative cyclization to afford the pyrido[4,3-b]carbazole framework proved to be very difficult [26]. Several attempts to optimize this reaction failed, which included using different reaction temperatures, different solvents (HOAc, HOPiv, dioxane, toluene), catalytic amounts of palladium(II) acetate in the presence of different re-oxidants, as well as stoichiometric amounts of palladium(II) acetate [27-29]. All of these experiments resulted to a large extent in decomposition, and led to olivacine (1) in only low to moderate yields with poor reproducibility.

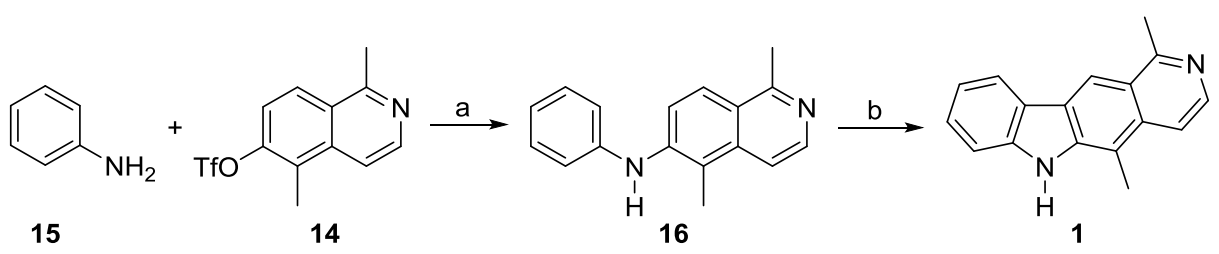

Scheme 3. Synthesis of olivacine (1) via oxidative cyclization. Reagents and conditions: (a) cat. $\mathrm{Pd}(\mathrm{OAc})_{2}$, cat. XPhos, $\mathrm{Cs}_{2} \mathrm{CO}_{3}, \mathrm{PhMe}$, reflux, $48 \mathrm{~h}, 100 \%$; (b) 1.1 equiv. $\mathrm{Pd}(\mathrm{OAc})_{2}, \mathrm{AcOH}, 80-100{ }^{\circ} \mathrm{C}, 24 \mathrm{~h}$, argon, $9-49 \%$.

Therefore, we decided to apply a Heck-type cyclization for the formation of the crucial carbon-carbon bond of the central pyrrole ring. This approach was already described by Sakamoto et al. in 1999 [30]. Buchwald-Hartwig coupling of the triflate 14 with the commercially available $o$-chloroanilines $\mathbf{1 7} \mathbf{a}-\mathbf{c}$ led to the corresponding diarylamines 18a-c in 83-94\% yield (Scheme 4). Compound 18a was structurally confirmed by an X-ray analysis (Figure 2).

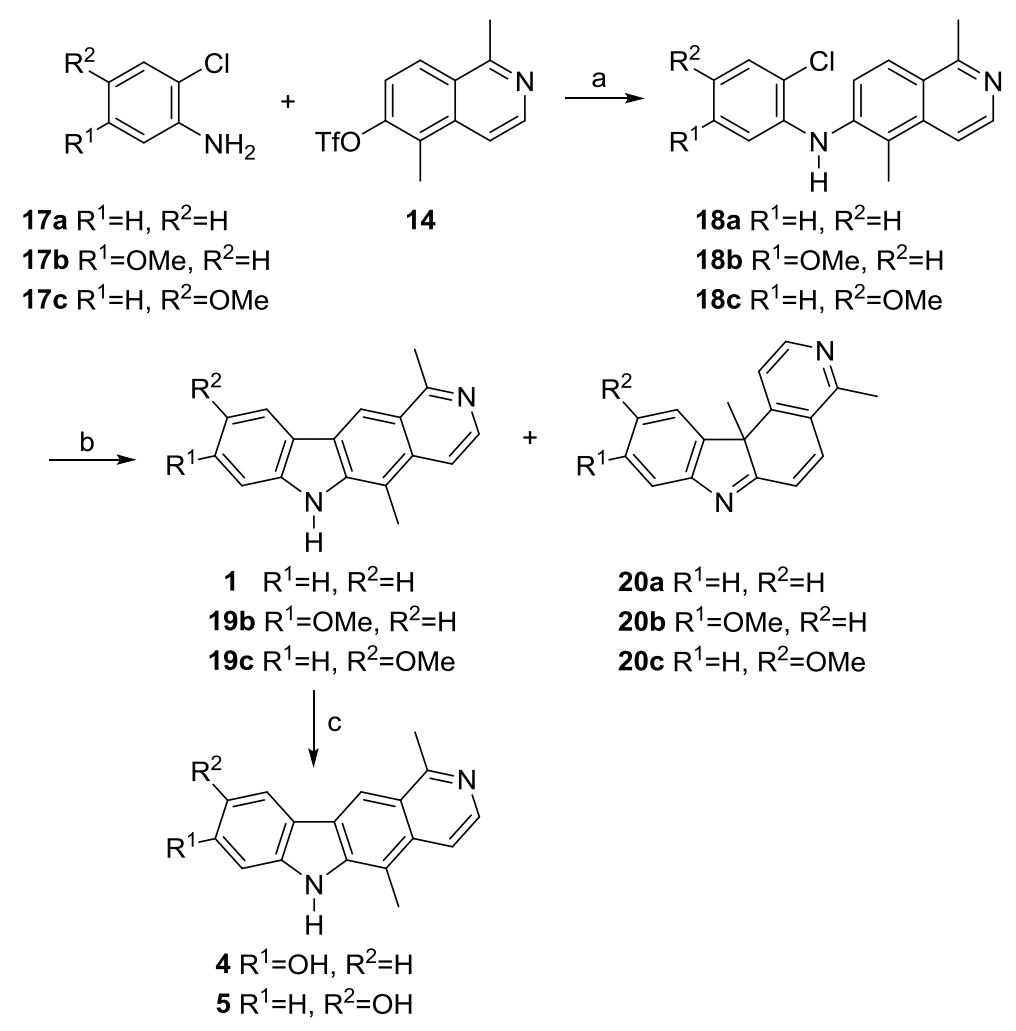

Scheme 4. Synthesis of the pyrido[4,3-b]carbazoles 1, 4 and 5. Reagents and conditions: (a) cat. $\mathrm{Pd}(\mathrm{OAc})_{2}$, cat. XPhos, $\mathrm{Cs}_{2} \mathrm{CO}_{3}, \mathrm{PhMe}$, reflux, 1-5 h, 83-94\% (18a-c); (b) cat. $\mathrm{Pd}(\mathrm{OAc})_{2}, \mathrm{P}(t \mathrm{Bu})_{3} \cdot \mathrm{HBF}_{4}, \mathrm{~K}_{2} \mathrm{CO}_{3}$, DMF, $140{ }^{\circ} \mathrm{C}, 20-35 \mathrm{~min}, 62-71 \%$ (1, 19b, 19c), 3-12\% (20a-c); (c) $\operatorname{HBr}_{(\mathrm{aq})}$, reflux, $24 \mathrm{~h}, 70-84 \%(4,5)$. 


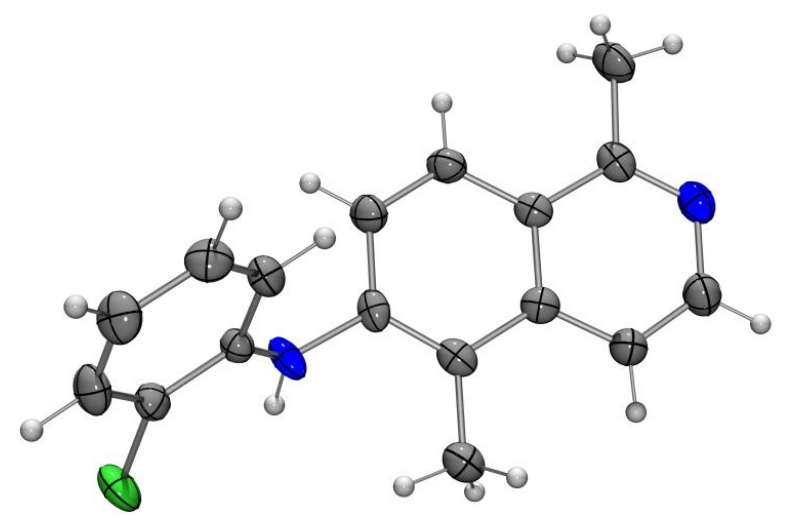

Figure 2. Molecular structure of the diarylamine 18a in the crystal (ORTEP plot showing thermal ellipsoids at the $50 \%$ probability level).

The cyclization reaction of the diarylamine 18a with catalytic amounts of palladium(II) acetate in the presence of $\mathrm{P}(t \mathrm{Bu})_{3} \cdot \mathrm{HBF}_{4}$ and $\mathrm{K}_{2} \mathrm{CO}_{3}$ in DMA at $110{ }^{\circ} \mathrm{C}$ or in DMF at $120{ }^{\circ} \mathrm{C}[31,32]$ proceeded very slowly and gave the product in only moderate yields after 1-2 days (Table 1, entries 1 and 4 ). Hydrodehalogenation leading to compound 16 was the major side reaction. Using only slightly higher temperatures $\left(130-140{ }^{\circ} \mathrm{C}\right)$, the reaction proceeded much faster, and the yields for olivacine (1) were significantly better (Table 1, entries 2, 5, and 6). Finally, using larger amounts of the catalyst combined with shorter reaction times, olivacine (1) was obtained in $71 \%$ yield. The structure of $\mathbf{1}$ was confirmed by an X-ray crystal structure determination (Figure 3).

Table 1. Optimization of the Heck-type cyclization of 18a to olivacine (1).

\begin{tabular}{ccccccccc}
\hline & Pd(OAc) $_{2}$ (Equiv.) & Ligand $^{\mathbf{1}}$ (Equiv.) & $\mathbf{K}_{\mathbf{2}} \mathbf{C O}_{\mathbf{3}}$ (Equiv.) & Solvent & Temp. $\left({ }^{\circ} \mathbf{C}\right)$ & Time (h) & Yield (\%) & RSM $^{2}$ (\%) \\
\hline 1 & 0.1 & 0.2 & 2 & DMA & 110 & 24 & 11 \\
2 & 0.1 & 0.2 & 2 & DMA & 130 & 1.5 & 46 \\
3 & 0.2 & 0.4 & 4 & DMA & 120 & 3.0 & 35 \\
$4^{3}$ & 0.5 & 1.0 & 10 & DMF & 120 & 45 & 46 & 35 \\
5 & 0.2 & 0.4 & 4 & DMF & 140 & 3.0 & 62 & 7 \\
6 & 0.3 & 0.6 & 4 & DMF & 140 & 0.5 & 71 \\
\hline
\end{tabular}

${ }^{1} \mathrm{P}(t \mathrm{Bu})_{3} \cdot \mathrm{HBF}_{4}$ was used as ligand; ${ }^{2} \mathrm{RSM}=$ reisolated starting material; ${ }^{3}$ reagents added in portions of 0.1 equiv. $\mathrm{Pd}(\mathrm{OAc})_{2}, 0.2$ equiv. ligand, 2 equiv. $\mathrm{K}_{2} \mathrm{CO}_{3}$ after $0,1,3,6,30 \mathrm{~h}$ of reaction time.

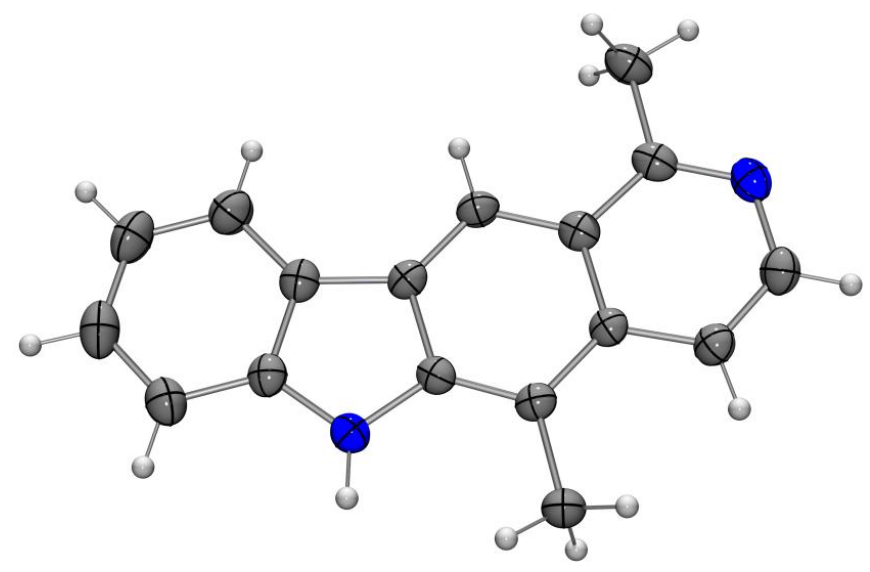

Figure 3. Molecular structure of olivacine (1) in the crystal (ORTEP plot showing thermal ellipsoids at the $50 \%$ probability level).

Application of these conditions to the cyclization of the diarylamines $\mathbf{1 8 b}$ and $\mathbf{1 8 c}$ provided 8-methoxyolivacine (19b) and 9-methoxyolivacine (19c) in 65\% and 62\% yield, respectively (Scheme 4). 
The structure of 8-methoxyolivacine $(\mathbf{1 9 b})$ was additionally confirmed by an X-ray analysis of single crystals (Figure 4). 9-Methoxyolivacine (19c) is a natural product that was isolated in 1967 from the bark of the coastal Venezuelan tree Aspidosperma vargasii A. DC. [33], and has been synthesized previously $[3,13,20]$. Interestingly, the $11 \mathrm{bH}$-pyrido[3,4-c]carbazoles $\mathbf{2 0 a}-\mathbf{c}$ containing a quaternary carbon atom were obtained as byproducts of the cyclization reactions of the diarylamines 18a-c in up to $12 \%$ yield. The structural assignments for the $11 \mathrm{bH}$-pyrido[3,4-c]carbazoles $20 \mathrm{a}-\mathrm{c}$ were supported by two-dimensional (2D) NMR (COSY, HMBC, HSQC, NOESY) spectroscopic studies (see Supplementary Materials). The compounds $20 \mathrm{a}-\mathrm{c}$ result from an attack at the $\mathrm{C} 5$ carbon atom of the isoquinoline moiety. Cleavage of the methyl ether of $\mathbf{1 9 b}$ and $\mathbf{1 9 c}$ provided 8-hydroxyolivacine (4) and 9-hydroxyolivacine (5) [3] in $84 \%$ and $70 \%$ yield, respectively. For biological testing, the products were additionally purified by HPLC.

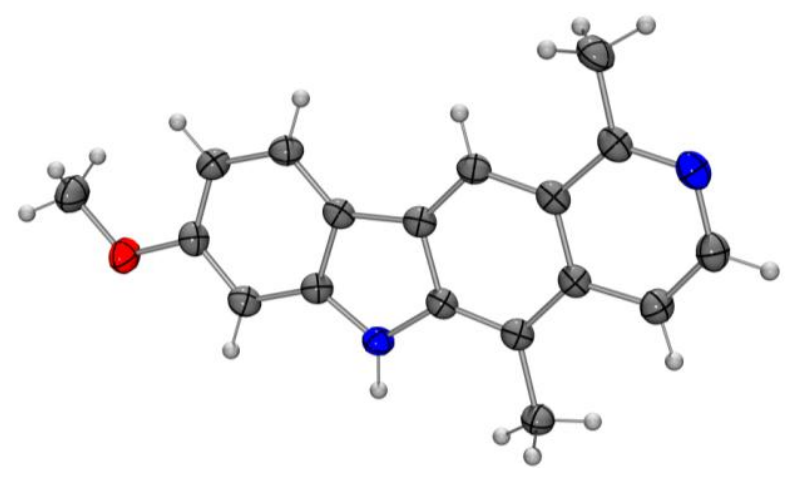

Figure 4. Molecular structure of 8-methoxyolivacine (19b) in the crystal (ORTEP plot showing thermal ellipsoids at the $50 \%$ probability level).

\subsection{Biological Activity}

A weak inhibitory activity against Mycobacterium tuberculosis was described in early reports for some simple tricyclic carbazole alkaloids [34-36]. Based on that work, we investigated the inhibitory activity of a range of oxygenated carbazole alkaloids and their derivatives, and found very promising results for several compounds [37-39]. Therefore, we also tested olivacine (1) and its oxygenated derivatives $4,5, \mathbf{1 9 b}$, and $19 \mathrm{c}$ for their inhibition of $M$. tuberculosis (Table 2).

Table 2. Inhibitory activity against $M$. tuberculosis of olivacine (1) and its oxygenated derivatives 4, 5, $19 b$, and 19c.

\begin{tabular}{cccc}
\hline Compound & MIC $_{\mathbf{9 0}}(\mu \mathbf{M})^{\mathbf{1}}$ & IC $_{\mathbf{5 0}}(\mu \mathbf{M})^{2}$ & SI $^{\mathbf{3}}$ \\
\hline Olivacine (1) & 4.7 & 18.05 & 3.8 \\
8-Hydroxyolivacine (4) & n.d. & n.d. & - \\
9-Hydroxyolivacine (5) & n.d. & n.d. & - \\
8-Methoxyolivacine (19b) & n.d. & n.d. & - \\
9-Methoxyolivacine (19c) $^{4}$ & 1.5 & 24.5 & 16.3 \\
3-Methoxy-2-methyl- $^{\text {(-Marbazole-1,4-quinone }}{ }^{5}$ & 4.0 & $>50$ & $>12.5$ \\
Isoniazid $^{5}$ & 0.24 & $>50$ & $>208$ \\
Rifampicin $^{5}$ & 0.02 & $>50$ & $>2500$ \\
\hline
\end{tabular}

\footnotetext{
${ }^{1}$ Minimum inhibitory concentrations $(\mu \mathrm{M})$ against $M$. tuberculosis $\mathrm{H}_{37} \mathrm{Rv}$ in the microplate Alamar blue assay (MABA); values are the mean of three replicate experiments; $n . d .=$ not determined. ${ }^{2}$ Cytotoxicity corresponding to the concentration $(\mu \mathrm{M})$ effecting a $50 \%$ decrease in tetrazolium dye reduction by Vero cells (African green monkey kidney cells); values are the mean of three replicate experiments; for experiments giving a value higher than the max. concentration used, $>50 \mu \mathrm{M}$ is denoted. ${ }^{3}$ Selectivity index: $\mathrm{SI}=\mathrm{IC}_{50} / \mathrm{MIC}_{90} .{ }^{4}$ These compounds showed no significant inhibition in a preliminary assay. ${ }^{5}$ 3-Methoxy-2-methylcarbazole-1,4-quinone, isoniazid, and rifampicin (rifampin) were used as positive control; solvent was used as negative control.
} 
In a preliminary activity test against $M$. tuberculosis, only two of the five pyrido[4,3- $b$ ]carbazoles, namely olivacine (1) and 9-methoxyolivacine (19c), showed significant effects and have been studied further. The minimum concentrations effecting a $90 \%$ inhibition of growth ( $\mathrm{MIC}_{90}$ ) of the M. tuberculosis strain $\mathrm{H}_{37} \mathrm{Rv}$ were determined by the microplate Alamar blue assay (MABA) [40,41]. The in vitro cytotoxicity towards mammalian (vero) cells was determined as described previously [40,42].

The $\mathrm{MIC}_{90}$ value for 3-methoxy-2-methylcarbazole-1,4-quinone served as a benchmark for comparison with the inhibitory activities of carbazoles that were found in our previous studies [39]. Although olivacine (1) shows an activity comparable to our benchmark compound, the SI value is considerably lower (SI = 3.8) due to its toxicity. However, 9-methoxyolivacine (19c) exhibits a strong inhibition of $M$. tuberculosis $\left(\mathrm{MIC}_{90}=1.5 \mu \mathrm{M}\right)$ combined with a lower cytotoxicity towards mammalian cells, which leads to a very good selectivity index $(\mathrm{SI}=16.3)$.

\section{Materials and Methods}

\subsection{General}

All of the reactions were carried out in oven-dried glassware using anhydrous solvents under an argon atmosphere, unless stated otherwise. $\mathrm{CH}_{2} \mathrm{Cl}_{2}, \mathrm{THF}$, and toluene were dried using a solvent purification system (MB-SBS, M. Braun Inertgas-Systeme GmbH, Garching, Germany). The petroleum ether that was used refers to the hydrocarbon mixture with a boiling range of $40-65{ }^{\circ} \mathrm{C} . \mathrm{Pd}(\mathrm{OAc})_{2}$ was recrystallized from glacial $\mathrm{AcOH}$. All of the other chemicals were used as received from commercial sources. A microwave reactor (CEM Discover, CEM GmbH, Kamp-Lintfort, Germany) was utilized for reactions taking place under microwave irradiation. Flash chromatography was performed using silica gel (0.035-0.070 mm) from Acros Organics (Thermo Fisher Scientific, Waltham, MA, USA). Alox N (aluminum oxide 90 active neutral) was obtained from Merck (Merck KGaA, Darmstadt, Germany). TLC was performed with TLC plates (silica gel $60 \mathrm{~F}_{254}$ ) from Merck (Merck KGaA, Darmstadt, Germany) using UV light for visualization. Melting points were measured on a Gallenkamp MPD 350 melting point apparatus (A. Gallenkamp \& Co. Ltd, London, UK). Ultraviolet spectra were recorded on a PerkinElmer $25 \mathrm{UV} /$ Vis spectrometer (Perkin Elmer, Waltham, MA, USA). Fluorescence spectra were obtained using a Varian Cary Eclipse spectrometer (Agilent, Santa Clara, CA, USA). IR spectra were recorded on a Thermo Nicolet Avatar 360 FT-IR spectrometer (Thermo Fisher Scientific, Waltham, MA, USA) using the ATR method (Attenuated Total Reflectance). NMR spectra were recorded on Bruker DRX 500 (Bruker Corp., Billerica, MA, USA) and Avance III 600 (Bruker Corp., Billerica, MA, USA) spectrometers. Chemical shifts $\delta$ are reported in parts per million (ppm) with the solvent signal as an internal standard. Standard abbreviations were used to denote the multiplicities of the signals. MS and HRMS (EI) were recorded on a Finnigan MAT-95 spectrometer (electron impact, $70 \mathrm{eV}$, Thermo Fisher Scientific, Waltham, MA, USA) or by GC/MS-coupling using an Agilent Technologies 6890 N GC System equipped with a 5973 Mass Selective Detector (electron impact, $70 \mathrm{eV}$, Agilent Technologies, Santa Clara, CA, USA). ESI-MS spectra were recorded on an Esquire LC with an ion trap detector from Bruker (Bruker Corp., Billerica, MA, USA). Positive and negative ions were detected. ESI-HRMS were recorded using a Q-TOF 6538 (Agilent Technologies, Santa Clara, CA, USA). Elemental analyses were measured on an EuroVector EuroEA3000 elemental analyzer (Eurovector Srl, Pavia, Italy). X-ray crystal structure analyses were performed with a Bruker-Nonius Kappa CCD (Bruker Corp., Billerica, MA, USA) that was equipped with a 700 series Cryostream low-temperature device from Oxford Cryosystems (Oxford Cryosystems Ltd, Long Hanborough, UK). SHELXS-97 [43], SADABS version 2.10 [44], SHELXL-97 [45], POV-Ray for Windows version 3.7.0.msvc10.win64 (Persistence of Vision Raytracer Pty. Ltd, Williamstown, Australia), and ORTEP-3 for Windows [46] (University of Glasgow, Glasgow, UK) were used as software. 


\subsection{Procedures}

1-Methoxy-2-methyl-3-(2-nitrovinyl)benzene. Nitromethane $(427 \mathrm{mg}, 6.99 \mathrm{mmol})$ and freshly sublimated ammonium acetate ( $433 \mathrm{mg}, 5.62 \mathrm{mmol}$ ) were added to a solution of 3-methoxy-2-methylbenzaldehyde (11, $800 \mathrm{mg}, 5.33 \mathrm{mmol})$ in acetic acid $(645 \mathrm{mg}, 10.74 \mathrm{mmol})$, and the mixture was stirred at $80{ }^{\circ} \mathrm{C}$ for $1 \mathrm{~h} 50 \mathrm{~min}$. After cooling to room temperature, the precipitate was dissolved by adding ethyl acetate. The mixture was transferred to a separatory funnel, and then washed twice with water and brine. The aqueous layer was extracted with ethyl acetate, the combined organic layers were dried (magnesium sulfate), and the solvent was evaporated. Purification of the residue by column chromatography (silica gel, petroleum ether/ethyl acetate, $1 \%$ to $15 \%$ ethyl acetate) provided 1-methoxy-2-methyl-3-(2-nitrovinyl)benzene (791 mg, $4.09 \mathrm{mmol}, 77 \%$ ) as yellow crystals. M.p. $97-98^{\circ} \mathrm{C}$; $\mathrm{UV}(\mathrm{MeOH}): \lambda=205,228,251,317 \mathrm{~nm}$; IR (ATR): $v=3116,2959,2920,2838,1901,1820,1697,1653$, 1627, 1594, 1573, 1541, 1498, 1477, 1450, 1331, 1260, 1244, 1102, 1080, 1007, 968, 893, 873, 844, 806, $777,725,693 \mathrm{~cm}^{-1} ;{ }^{1} \mathrm{H}-\mathrm{NMR}\left(500 \mathrm{MHz}, \mathrm{CDCl}_{3}\right): \delta=2.33(\mathrm{~s}, 3 \mathrm{H}), 3.86(\mathrm{~s}, 3 \mathrm{H}), 6.96(\mathrm{~d}, J=8.2 \mathrm{~Hz}, 1 \mathrm{H})$, $7.10(\mathrm{~d}, J=7.8 \mathrm{~Hz}, 1 \mathrm{H}), 7.22(\mathrm{t}, J=8.0 \mathrm{~Hz}, 1 \mathrm{H}), 7.48(\mathrm{~d}, J=13.5 \mathrm{~Hz}, 1 \mathrm{H}), 8.33(\mathrm{~d}, J=13.5 \mathrm{~Hz}, 1 \mathrm{H})$; ${ }^{13} \mathrm{C}-\mathrm{NMR}\left(125 \mathrm{MHz}, \mathrm{CDCl}_{3}\right): \delta=11.96\left(\mathrm{CH}_{3}\right), 55.84\left(\mathrm{CH}_{3}\right), 113.12(\mathrm{CH}), 119.25(\mathrm{CH}), 127.11(\mathrm{CH})$, 128.40 (C), 130.13 (C), 137.25 (CH), 138.20 (CH), 158.29 (C); MS (EI): $m / z(\%)=193$ (100, [M] $\left.]^{+}\right), 178(6)$, 161 (7), 146 (70), 131 (52), 115 (54), 103 (67), 91 (37), 77 (47), 63 (18), 51 (18); HRMS: calcd. for $\mathrm{C}_{10} \mathrm{H}_{11} \mathrm{NO}_{3}$ : 193.0738, found: 193.0733; elemental analysis: calcd. for $\mathrm{C}_{10} \mathrm{H}_{11} \mathrm{NO}_{3}$ : C: 62.17, H: 5.74, N: 7.25; found: C: $62.16, \mathrm{H}: 5.77, \mathrm{~N}: 7.50$.

2-(3-Methoxy-2-methylphenyl)ethanamine. Over a period of $1 \mathrm{~h}$, a solution of 1-methoxy-2-methyl-3(2nitrovinyl)benzene $(6.79 \mathrm{~g}, 35.2 \mathrm{mmol})$ in THF $(95 \mathrm{~mL})$ was added to a suspension of lithium aluminum hydride $(6.83 \mathrm{~g}, 180 \mathrm{mmol})$ in THF $(360 \mathrm{~mL})$ at $0{ }^{\circ} \mathrm{C}$. The cooling bath was removed, and the mixture was heated for $30 \mathrm{~min}$ at room temperature and $18 \mathrm{~h}$ at reflux. A second portion of lithium aluminum hydride $(0.35 \mathrm{~g}, 9.1 \mathrm{mmol})$ was added to the slightly reddish-colored solution, and the mixture was heated at reflux for an additional hour. After cooling to room temperature, the reaction mixture was carefully quenched with saturated aqueous ammonium chloride, and the $\mathrm{pH}$ value was adjusted to nine. Diethyl ether was added, and the mixture was transferred into a separatory funnel. Still under argon, the layers were separated, and the aqueous layer was extracted three times with diethyl ether. The combined organic layers were washed with water and brine, dried (magnesium sulfate), and the solvent was evaporated to provide 2-(3-methoxy-2-methylphenyl)ethanamine (5.33 g, $32.3 \mathrm{mmol}, 92 \%)$ as a yellow oil. UV (MeOH): $\lambda=204,218,273,280 \mathrm{~nm}$; IR (ATR): $v=3402,2989,2923,2848,2659,2480$, 2065, 1658, 1604, 1581, 1510, 1463, 1395, 1293, 1256, 1194, 1171, 1149, 1122, 1096, 1006, 953, 875, 789, $776,763,719 \mathrm{~cm}^{-1},{ }^{1} \mathrm{H}-\mathrm{NMR}\left(600 \mathrm{MHz}, \mathrm{CDCl}_{3}\right): \delta=2.22(\mathrm{~s}, 3 \mathrm{H}), 3.10-3.19(\mathrm{~m}, 4 \mathrm{H}), 3.82(\mathrm{~s}, 3 \mathrm{H}), 6.77$ $(\mathrm{d}, J=8.2 \mathrm{~Hz}, 1 \mathrm{H}), 6.83(\mathrm{~d}, J=7.6 \mathrm{~Hz}, 1 \mathrm{H}), 7.13(\mathrm{t}, J=7.9 \mathrm{~Hz}, 1 \mathrm{H}) ;{ }^{13} \mathrm{C}-\mathrm{NMR}\left(150 \mathrm{MHz}\right.$, methanol- $\left.d_{4}\right)$ : $\delta=11.47\left(\mathrm{CH}_{3}\right), 32.26\left(\mathrm{CH}_{2}\right), 40.35\left(\mathrm{CH}_{2}\right), 55.49\left(\mathrm{CH}_{3}\right), 109.10(\mathrm{CH}), 121.76(\mathrm{CH}), 125.07(\mathrm{C}), 126.59$ $(\mathrm{CH}), 135.84(\mathrm{C}), 158.03(\mathrm{C})$; MS (ESI, $+10 \mathrm{~V}): \mathrm{m} / z=149.0\left[\mathrm{M}-\mathrm{NH}_{3}+\mathrm{H}\right]^{+}, 166.0[\mathrm{M}+\mathrm{H}]^{+}, 331.2[2 \mathrm{M}+$ $\mathrm{H}]^{+}$; HRMS: calcd. for $\mathrm{C}_{10} \mathrm{H}_{15} \mathrm{NO}$ : 165.1153, found: 165.1144 .

2-(3-Methoxy-2-methylphenyl)ethyl acetamide (12). DMAP (14 $\mathrm{mg}, 0.11 \mathrm{mmol})$ was added to a solution of 2-(3-methoxy-2-methylphenyl)ethanamine $(230 \mathrm{mg}, 1.14 \mathrm{mmol})$ in pyridine $(4.5 \mathrm{~mL})$, and the mixture was cooled to $0{ }^{\circ} \mathrm{C}$. Acetic anhydride $(140 \mu \mathrm{L}, 15 \mathrm{mmol})$ was added dropwise over a period of five minutes, and the reaction mixture was stirred for $4 \mathrm{~h}$. The solvent was evaporated, and the raw material was purified by chromatography (Alox N, $5 \% \mathrm{H}_{2} \mathrm{O}$; ethyl acetate) to provide 2-(3-methoxy-2-methylphenyl)ethyl acetamide $(\mathbf{1 2}, 235 \mathrm{mg}, 1.13 \mathrm{mmol}, 99 \%)$ as a light yellow solid. M.p. 84-85 ${ }^{\circ} \mathrm{C}$; UV (MeOH): $\lambda=205,219,229,271,279$ nm; IR (ATR): $v=3267,3085,2932,2836,2030$, 2009, 1976, 1716, 1659, 1630, 1564, 1508, 1489, 1472, 1459, 1435, 1396, 1370, 1298, 1285, 1247, 1201, 1180, 1110, 1092, 1037, 1013, 896, 812, 776, 748, 723, 701, 651, $606 \mathrm{~cm}^{-1} ;{ }^{1} \mathrm{H}-\mathrm{NMR}\left(500 \mathrm{MHz}, \mathrm{CDCl}_{3}\right): \delta=1.95$ $(\mathrm{s}, 3 \mathrm{H}), 2.19(\mathrm{~s}, 3 \mathrm{H}), 2.84(\mathrm{t}, J=6.9 \mathrm{~Hz}, 2 \mathrm{H}), 3.46(\mathrm{q}, J=6.9 \mathrm{~Hz}, 2 \mathrm{H}), 3.82(\mathrm{~s}, 3 \mathrm{H}), 5.46(\mathrm{br} \mathrm{s}, 1 \mathrm{H}), 6.76$ $(\mathrm{d}, J=7.9 \mathrm{~Hz}, 2 \mathrm{H}), 7.12(\mathrm{t}, J=7.9 \mathrm{~Hz}, 1 \mathrm{H}) ;{ }^{13} \mathrm{C}-\mathrm{NMR}\left(125 \mathrm{MHz}, \mathrm{CDCl}_{3}\right): \delta=11.53\left(\mathrm{CH}_{3}\right), 23.51\left(\mathrm{CH}_{3}\right)$, 
$33.38\left(\mathrm{CH}_{2}\right), 39.87\left(\mathrm{CH}_{2}\right), 55.64\left(\mathrm{CH}_{3}\right), 108.61(\mathrm{CH}), 121.89(\mathrm{CH}), 125.24(\mathrm{C}), 126.36(\mathrm{CH}), 138.38(\mathrm{C})$, $158.09(\mathrm{C}), 170.21(\mathrm{C}=\mathrm{O})$; $\mathrm{MS}(\mathrm{ESI},+10 \mathrm{~V}): m / z=208.0[\mathrm{M}+\mathrm{H}]^{+}, 415.1[2 \mathrm{M}+\mathrm{H}]^{+}, 437.1[2 \mathrm{M}+\mathrm{Na}]^{+}$; HRMS: calcd. for $\mathrm{C}_{12} \mathrm{H}_{17} \mathrm{NO}_{2}$ : 207.1259, found: 207.1248; elemental analysis: calcd. for $\mathrm{C}_{12} \mathrm{H}_{17} \mathrm{NO}_{2}$ : $\mathrm{C}_{\text {: }}$ 69.54, H: 8.27, N: 6.76; found: C: 69.04, H: 8.73, N: 6.78 .

6-Methoxy-1,5-dimethyl-3,4-dihydroisoquinoline. Phosphorus oxychloride (1.9 mL, $21 \mathrm{mmol})$ was added to a refluxing solution of acetamide $12(433 \mathrm{mg}, 2.09 \mathrm{mmol})$ in freshly distilled chloroform $(23 \mathrm{~mL})$, and the mixture was stirred for one hour. Subsequently, solvent and excess phosphorus oxychloride were removed under vigorous stirring by a nitrogen stream through a pair of soda lye-filled gas washing bottles. The remaining oily raw product was dissolved in ethyl acetate. Soda lye (10\%) was added, and the $\mathrm{pH}$ value was adjusted to 8-9 using saturated aqueous ammonium chloride. The layers were separated, and the aqueous layer was extracted with ethyl acetate. The combined organic layers were washed with water and brine, and then dried (magnesium sulfate), and the solvent was evaporated. Purification of the crude product by chromatography (Alox N, 5\% $\mathrm{H}_{2} \mathrm{O}$; ethyl acetate $+3 \%$ triethylamine) afforded 6-methoxy-1,5-dimethyl-3,4-dihydroisoquinoline (393 mg, $2.08 \mathrm{mmol}, 99 \%$ ) as a yellow solid. M.p. $57-58{ }^{\circ} \mathrm{C}$ (subl.); UV (MeOH): $\lambda=229,274,319 \mathrm{~nm}$; IR (ATR): $v=3002,2939,2838,1735,1699,1629,1594,1576,1539,1507,1482,1435,1368,1291,1258,1184,1149$, 1101, 1015, 922, 901, 873, 805, 751, 700, 666, $637 \mathrm{~cm}^{-1} ;{ }^{1} \mathrm{H}-\mathrm{NMR}\left(500 \mathrm{MHz}, \mathrm{CDCl}_{3}\right): \delta=2.15(\mathrm{~s}, 3 \mathrm{H}), 2.35$ $(\mathrm{t}, J=1.4 \mathrm{~Hz}, 3 \mathrm{H}), 2.64(\mathrm{t}, J=7.4 \mathrm{~Hz}, 2 \mathrm{H}), 3.63(\mathrm{tq}, J=7.4,1.4 \mathrm{~Hz}, 2 \mathrm{H}), 3.85(\mathrm{~s}, 3 \mathrm{H}), 6.75(\mathrm{~d}, J=8.5 \mathrm{~Hz}, 1 \mathrm{H})$, $7.37(\mathrm{~d}, J=8.5 \mathrm{~Hz}, 1 \mathrm{H}) ;{ }^{13} \mathrm{C}-\mathrm{NMR}\left(125 \mathrm{MHz}, \mathrm{CDCl}_{3}\right): \delta=11.08\left(\mathrm{CH}_{3}\right), 23.32\left(\mathrm{CH}_{2}\right), 23.62\left(\mathrm{CH}_{3}\right), 46.91$ $\left(\mathrm{CH}_{2}\right), 55.61\left(\mathrm{CH}_{3}\right), 107.50(\mathrm{CH}), 123.06(\mathrm{C}), 123.40(\mathrm{C}), 124.76(\mathrm{CH}), 137.70(\mathrm{C}), 159.47(\mathrm{C}), 164.80(\mathrm{C})$; MS (EI): $m / z(\%)=189\left(95,[\mathrm{M}]^{+}\right), 174(100), 158(16), 144$ (23), 131 (22), 115 (31), 105 (23), 91 (22), 77 (29), 63 (17), 51 (20); HRMS: calcd. for $\mathrm{C}_{12} \mathrm{H}_{15} \mathrm{NO}$ : 189.1154, found: 189.1147; elemental analysis: calcd. for $\mathrm{C}_{12} \mathrm{H}_{15} \mathrm{NO}: \mathrm{C}: 76.16, \mathrm{H}: 7.99, \mathrm{~N}: 7.40$; found: C: 76.25, H: 7.98, N: 7.46.

6-Methoxy-1,5-dimethylisoquinoline (13). A flask filled with 6-methoxy-1,5-dimethyl-3,4-dihydroisoquinoline (90.3 $\mathrm{mg}, 0.48 \mathrm{mmol})$ and palladium on charcoal $(10 \%, 93.6 \mathrm{mg})$ was evacuated under vigorous stirring for $15 \mathrm{~min}$, and then filled with argon. Toluene $(3.6 \mathrm{~mL})$ and cyclohexene $(1.3 \mathrm{~mL}, 13 \mathrm{mmol})$ were added, and the mixture was heated at reflux until full conversion was detected (TLC: Alox N; ethyl acetate/isohexane, 2:1 + 1 drop of ethanol). The catalyst was removed by filtration (ethyl acetate), and the crude product was purified by chromatography (Alox $\mathrm{N}, 5 \% \mathrm{H}_{2} \mathrm{O}$; petroleum ether/ethyl acetate, 5:1) to provide 6-methoxy-1,5-dimethylisoquinoline (13, $90 \mathrm{mg}, 0.48 \mathrm{mmol}, 100 \%)$ as a beige solid. M.p. 99-102 ${ }^{\circ} \mathrm{C}$; UV (MeOH): $\lambda=203,236,301$ nm; IR (ATR): $v=3058,3015,2965,2940,2847$, 1608, 1563, 1542, 1495, 1457, 1401, 1344, 1324, 1267, 1179, 1153, 1116, 1078, 1009, 984, 913, 848, 814, 774, 698, 673, 648, 581, $528 \mathrm{~cm}^{-1},{ }^{1} \mathrm{H}-\mathrm{NMR}\left(600 \mathrm{MHz}^{\mathrm{C}} \mathrm{CDCl}_{3}\right): \delta=2.50(\mathrm{~s}, 3 \mathrm{H}), 2.97(\mathrm{~s}, 3 \mathrm{H}), 4.00(\mathrm{~s}, 3 \mathrm{H}), 7.35$ $(\mathrm{d}, J=9.2 \mathrm{~Hz}, 1 \mathrm{H}), 7.63(\mathrm{~d}, J=6.2 \mathrm{~Hz}, 1 \mathrm{H}), 8.05(\mathrm{~d}, J=9.2 \mathrm{~Hz}, 1 \mathrm{H}), 8.32(\mathrm{~d}, J=6.2 \mathrm{~Hz}, 1 \mathrm{H}) ;{ }^{13} \mathrm{C}-\mathrm{NMR}$ $\left(150 \mathrm{MHz}, \mathrm{CDCl}_{3}\right): \delta=10.49\left(\mathrm{CH}_{3}\right), 22.17\left(\mathrm{CH}_{3}\right), 56.39\left(\mathrm{CH}_{3}\right), 113.71(\mathrm{CH}), 115.83(\mathrm{CH}), 118.97(\mathrm{C})$, $123.04(\mathrm{C}), 125.66(\mathrm{CH}), 127.47(\mathrm{C}), 136.96(\mathrm{C}), 140.72(\mathrm{CH}), 158.57(\mathrm{C}) ; \mathrm{MS}(\mathrm{EI}): \mathrm{m} / z(\%)=187(100$, $\left.[\mathrm{M}]^{+}\right), 172$ (26), 156 (16), 144 (80), 128 (19), 115 (43), 103 (21), 89 (11), 77 (30), 63 (24), 51 (22); HRMS: calcd. for $\mathrm{C}_{12} \mathrm{H}_{13} \mathrm{NO}$ : 187.0997 , found: 187.0986 ; elemental analysis: calcd. for $\mathrm{C}_{12} \mathrm{H}_{13} \mathrm{NO}$ : C: $76.98, \mathrm{H}$ : 7.00, N: 7.48; found: C: 76.43, H: 7.04, N: 7.53 .

1,5-Dimethylisoquinolin-6-ol. For small amounts: In a microwave tube, a mixture of 6-methoxy-1,5dimethylisoquinoline $(\mathbf{1 3}, 45 \mathrm{mg}, 0.24 \mathrm{mmol})$ and pyridinium chloride $(1 \mathrm{~g}, 8 \mathrm{mmol})$ was irradiated at $155{ }^{\circ} \mathrm{C}(300 \mathrm{~W})$ for $30 \mathrm{~min}$. After cooling to room temperature, the mixture was dissolved in water and ethyl acetate, and neutralized with a saturated aqueous solution of sodium bicarbonate. The layers were separated, and the aqueous layer was carefully extracted with ethyl acetate. The combined organic layers were dried (magnesium sulfate), and the solvent was evaporated to give 1,5-dimethylisoquinolin-6-ol (40 mg, $0.23 \mathrm{mmol}, 96 \%)$ as a brownish solid.

For larger amounts: Freshly distilled hydrobromic acid $(22 \mathrm{~mL}, 0.19 \mathrm{~mol})$ was carefully added at $0{ }^{\circ} \mathrm{C}$ to 6-methoxy-1,5-dimethylisoquinoline $(\mathbf{1 3}, 3.01 \mathrm{~g}, 16.1 \mathrm{mmol})$. After the addition was completed, 
the cooling bath was removed, and the mixture was heated at reflux for five hours. Then, the excess of hydrobromic acid was removed under vacuo. The brownish raw material was completely dissolved in water $(115 \mathrm{~mL}$, ultrasound), filtered, and neutralized by the dropwise addition of a saturated aqueous solution of sodium bicarbonate. The resulting solid was carefully washed with water and dried in vacuo to provide 1,5-dimethylisoquinolin-6-ol (2.49 $\mathrm{g}, 14.4 \mathrm{mmol}, 89 \%)$ as a brownish solid. M.p. 248-250 ${ }^{\circ} \mathrm{C}$ (sublimation); UV (MeOH): $\lambda=234,279,301,328,382 \mathrm{~nm}$; IR (ATR): $v=2920,2850,2475$ (br), 1808 (br), 1617, 1599, 1564, 1479, 1423, 1385, 1356, 1337, 1279, 1202, 1057, 1006, 939, 813, 774, 718, 672, $660 \mathrm{~cm}^{-1} ;{ }^{1} \mathrm{H}-\mathrm{NMR}\left(500 \mathrm{MHz}\right.$, methanol- $\left.d_{4}\right): \delta=2.43(\mathrm{~s}, 3 \mathrm{H}), 2.84(\mathrm{~s}, 3 \mathrm{H}), 7.22(\mathrm{~d}, J=9.1 \mathrm{~Hz}$, $1 \mathrm{H}), 7.64(\mathrm{~d}, J=6.2 \mathrm{~Hz}, 1 \mathrm{H}), 7.95(\mathrm{~d}, J=9.1 \mathrm{~Hz}, 1 \mathrm{H}), 8.12(\mathrm{~d}, J=6.2 \mathrm{~Hz}, 1 \mathrm{H})$; ${ }^{13} \mathrm{C}-\mathrm{NMR}(125 \mathrm{MHz}$, methanol- $\left.d_{4}\right): \delta=10.13\left(\mathrm{CH}_{3}\right), 21.33\left(\mathrm{CH}_{3}\right), 116.26(\mathrm{C}), 116.72(\mathrm{CH}), 119.91(\mathrm{CH}), 123.50(\mathrm{C}), 126.19$ (CH), 138.79 (C), 140.60 (CH), 157.91 (C), 158.92 (C); MS (ESI, +10 V): $m / z=174.0[\mathrm{M}+\mathrm{H}]^{+} ; \mathrm{HRMS}$ calcd. for $\mathrm{C}_{11} \mathrm{H}_{11} \mathrm{NO}$ : 173.0841, found: 173.0851; elemental analysis: calcd. for $\mathrm{C}_{11} \mathrm{H}_{11} \mathrm{NO}$ : C: $76.28, \mathrm{H}$ : 6.40, N: 8.09; found: C: 76.00, H: 6.47, N: 8.21.

1,5-Dimethylisoquinolin-6-yl trifluoromethanesulfonate (14). Pyridine (1.1 mL, $12 \mathrm{mmol})$ was added to a suspension of 1,5-dimethylisoquinolin-6-ol $(0.60 \mathrm{~g}, 3.5 \mathrm{mmol})$ in acetonitrile $(66 \mathrm{~mL})$ at $0{ }^{\circ} \mathrm{C}$. Subsequently, trifluoromethanesulfonic anhydride $(0.87 \mathrm{~mL}, 5.2 \mathrm{mmol})$ was added dropwise, and the reaction mixture was stirred at this temperature for $20 \mathrm{~h}$. Ethyl acetate and water were added, and the layers were separated. The aqueous layer was extracted three times with ethyl acetate. The combined organic layers were washed with water and brine, and then dried (sodium sulfate). The solvent was evaporated, and the residue was purified by column chromatography (silica gel, pentane/ethyl acetate, 1:1) to provide 1,5-dimethylisoquinolin-6-yl trifluoromethanesulfonate (14, $0.92 \mathrm{~g}, 3.0 \mathrm{mmol}, 87 \%)$ as a beige solid. M.p. $67-67.5^{\circ} \mathrm{C}$; UV (MeOH): $\lambda=198,219,274,308,321 \mathrm{~nm}$; IR (ATR): $v=3088,3031$, 2995, 2927, 2856, 1612, 1564, 1522, 1473, 1459, 1414, 1375, 1350, 1245, 1207, 1170, 1132, 1038, 994, 933, 861, 826, 815, 767, 663, $621 \mathrm{~cm}^{-1} ;{ }^{1} \mathrm{H}-\mathrm{NMR}\left(500 \mathrm{MHz}, \mathrm{CDCl}_{3}\right): \delta=2.68(\mathrm{~s}, 3 \mathrm{H}), 3.00(\mathrm{~s}, 3 \mathrm{H}), 7.48(\mathrm{~d}$, $J=9.3 \mathrm{~Hz}, 1 \mathrm{H}), 7.71(\mathrm{~d}, J=6.1 \mathrm{~Hz}, 1 \mathrm{H}), 8.10(\mathrm{~d}, J=9.3 \mathrm{~Hz}, 1 \mathrm{H}), 8.52(\mathrm{~d}, J=6.1 \mathrm{~Hz}, 1 \mathrm{H}) ;{ }^{13} \mathrm{C}-\mathrm{NMR}$ $\left(125 \mathrm{MHz}_{\mathrm{CDCl}}\right): \delta=12.24\left(\mathrm{CH}_{3}\right), 22.88\left(\mathrm{CH}_{3}\right), 116.23(\mathrm{CH}), 118.77\left(\mathrm{q}, J_{C, F}=321 \mathrm{~Hz}, \mathrm{CF}_{3}\right), 120.75$ $(\mathrm{CH}), 126.42\left(\mathrm{CH}\right.$ and C), $126.64(\mathrm{C}), 136.97(\mathrm{C}), 143.40(\mathrm{CH}), 147.91(\mathrm{C}), 159.50(\mathrm{C}) ;{ }^{19} \mathrm{~F}-\mathrm{NMR}(282 \mathrm{MHz}$, $\left.\mathrm{CDCl}_{3}\right): \delta=-73.58(\mathrm{~s}, 3 \mathrm{~F}) ; \mathrm{MS}(\mathrm{EI}): \mathrm{m} / z(\%)=305\left(1,[\mathrm{M}]^{+}\right), 172(8), 144(48), 128(7), 115(19), 103(13)$, 89 (5), 77 (18), 69 (100), 63 (10), 51 (12); MS (ESI, +10 V): $m / z=306.0[\mathrm{M}+\mathrm{H}]^{+}$; elemental analysis: calcd. for $\mathrm{C}_{12} \mathrm{H}_{10} \mathrm{~F}_{3} \mathrm{NO}_{3} \mathrm{~S}: \mathrm{C}: 47.21, \mathrm{H}: 3.30, \mathrm{~N}: 4.59$, S: 10.50; found: C: 47.09, H: 3.02, N: 4.58, S: 10.45 .

1,5-Dimethyl-N-phenylisoquinolin-6-amine (16). Aniline $(\mathbf{1 5}, 0.1 \mathrm{~mL}, 1.2 \mathrm{mmol})$ was added dropwise to a solution of 1,5-dimethylisoquinolin-6-yl trifluoromethanesulfonate (14, $0.235 \mathrm{~g}, 0.770 \mathrm{mmol})$, palladium(II) acetate $(13 \mathrm{mg}, 58 \mu \mathrm{mol}), \mathrm{XPhos}(55 \mathrm{mg}, 0.12 \mathrm{mmol})$, and cesium carbonate $(0.35 \mathrm{~g}, 1.1 \mathrm{mmol})$ in toluene $(20 \mathrm{~mL})$. The mixture was heated at reflux for $48 \mathrm{~h}$. After cooling to room temperature, the reaction mixture was filtered over a short pad of Hyflo (ethyl acetate), and the solvent was evaporated. Purification of the residue by column chromatography (silica gel, dichloromethane/ethyl acetate 1:3 $+1 \%$ methanol) provided 1,5-dimethyl- $N$-phenylisoquinolin-6-amine (16, $0.19 \mathrm{~g}, 0.77 \mathrm{mmol}, 100 \%)$ as a yellow solid. M.p. $175^{\circ} \mathrm{C}$ (decomp.); UV (MeOH): $\lambda=223,250,280,325,358$ (sh) nm; IR (ATR): $v=3207,3163,3090,3012,2985,2919,2860,1632,1615,1594,1562,1526,1492,1439,1397,1380,1310$, 1286, 1174, 1151, 1060, 990, 938, 864, 844, 819, 788, 748, 695, $678 \mathrm{~cm}^{-1} ;{ }^{1} \mathrm{H}-\mathrm{NMR}\left(500 \mathrm{MHz}, \mathrm{CDCl}_{3}\right)$ : $\delta=2.50(\mathrm{~s}, 3 \mathrm{H}), 2.91(\mathrm{~s}, 3 \mathrm{H}), 5.83($ br s, $1 \mathrm{H}), 7.01(\mathrm{t}, J=7.4 \mathrm{~Hz}, 1 \mathrm{H}), 7.05(\mathrm{~d}, J=7.7 \mathrm{~Hz}, 2 \mathrm{H}), 7.29-7.33(\mathrm{~m}$, $2 \mathrm{H}), 7.53(\mathrm{~d}, J=9.1 \mathrm{~Hz}, 1 \mathrm{H}), 7.60(\mathrm{~d}, J=6.1 \mathrm{~Hz}, 1 \mathrm{H}), 7.92(\mathrm{~d}, J=9.1 \mathrm{~Hz}, 1 \mathrm{H}), 8.35(\mathrm{~d}, J=6.1 \mathrm{~Hz}, 1 \mathrm{H})$; ${ }^{13} \mathrm{C}-\mathrm{NMR}\left(125 \mathrm{MHz}, \mathrm{CDCl}_{3}\right): \delta=12.38\left(\mathrm{CH}_{3}\right), 22.66\left(\mathrm{CH}_{3}\right), 115.32(\mathrm{CH}), 118.78(\mathrm{C}), 118.88(2 \mathrm{CH}), 119.96$ (CH), $121.99(\mathrm{CH}), 123.77(\mathrm{C}), 124.80(\mathrm{CH}), 129.63(2 \mathrm{CH}), 136.93(\mathrm{C}), 141.95(\mathrm{C}), 142.27(\mathrm{CH}), 142.91(\mathrm{C})$, 158.59 (C); MS (EI): $m / z(\%)=248\left(100,[\mathrm{M}]^{+}\right), 233(16), 171(17) ; \mathrm{MS}(\mathrm{ESI},+10 \mathrm{~V}): m / z=249.1[\mathrm{M}+\mathrm{H}]^{+}$; HRMS (ESI): calcd. for $\mathrm{C}_{17} \mathrm{H}_{16} \mathrm{~N}_{2}$ : 248.1313, found: 248.1310 .

N-(2-Chlorophenyl)-1,5-dimethylisoquinolin-6-amine (18a). 2-Chloroaniline (17a, $78 \mu \mathrm{L}, 0.74 \mathrm{mmol})$ was added dropwise to a solution of 1,5-dimethylisoquinolin-6-yl trifluoromethanesulfonate (14, $0.15 \mathrm{~g}$, $0.49 \mathrm{mmol})$, palladium(II) acetate $(8.3 \mathrm{mg}, 37 \mu \mathrm{mol})$, XPhos (35 mg, $74 \mu \mathrm{mol})$, and cesium carbonate 
(224 mg, $0.688 \mathrm{mmol})$ in toluene $(12 \mathrm{~mL})$. The mixture was heated at reflux for $1.5 \mathrm{~h}$. After cooling to room temperature, the reaction mixture was filtered over a short pad of Hyflo (ethyl acetate), and the solvent was evaporated. Purification of the residue by column chromatography (silica gel, dichloromethane/ethyl acetate, 9:1 to 0:1, each $+1 \%$ ethanol) provided $N$-(2-chlorophenyl)-1,5-dimethylisoquinolin-6-amine (18a, $0.130 \mathrm{~g}, 0.460 \mathrm{mmol}, 94 \%$ ) as brownish crystals. M.p. $194-198{ }^{\circ} \mathrm{C}$; UV (MeOH): $\lambda=221,249,278$, $320 \mathrm{~nm}$; fluorescence $(\mathrm{MeOH}): \lambda_{\mathrm{ex}}=221, \lambda_{\mathrm{em}}=229$ (sh), $334 \mathrm{~nm}$; IR (ATR): $v=3189,3078,2955,2919$, 2850, 1589, 1567, 1542, 1501, 1474, 1452, 1396, 1367, 1307, 1294, 1267, 1225, 1198, 1129, 1058, 1033, 996, 933, 862, 843, 822, 793, 751, $706 \mathrm{~cm}^{-1} ;{ }^{1} \mathrm{H}-\mathrm{NMR}\left(500 \mathrm{MHz}, \mathrm{CDCl}_{3}\right): \delta=2.35(\mathrm{~s}, 3 \mathrm{H}), 2.93(\mathrm{~s}, 3 \mathrm{H}), 6.15(\mathrm{br}$ $\mathrm{s}, 1 \mathrm{H}), 6.85(\mathrm{td}, J=7.7,3.0 \mathrm{~Hz}, 1 \mathrm{H}), 6.95(\mathrm{dd}, J=8.2,1.4 \mathrm{~Hz}, 1 \mathrm{H}), 7.10-7.14(\mathrm{~m}, 1 \mathrm{H}), 7.40(\mathrm{dd}, J=8.0$, $1.4 \mathrm{~Hz}, 1 \mathrm{H}), 7.51(\mathrm{~d}, J=9.0 \mathrm{~Hz}, 1 \mathrm{H}), 7.63(\mathrm{~d}, J=6.1 \mathrm{~Hz}, 1 \mathrm{H}), 7.96(\mathrm{~d}, J=9.0 \mathrm{~Hz}, 1 \mathrm{H}), 8.39(\mathrm{~d}, J=6.1 \mathrm{~Hz}$, $1 \mathrm{H}) ;{ }^{13} \mathrm{C}-\mathrm{NMR}\left(125 \mathrm{MHz}, \mathrm{CDCl}_{3}\right): \delta=12.58\left(\mathrm{CH}_{3}\right), 22.58\left(\mathrm{CH}_{3}\right), 115.47(\mathrm{CH}), 116.05(\mathrm{CH}), 120.90(\mathrm{CH})$, $121.87(\mathrm{C}), 122.07(\mathrm{CH}), 122.83(\mathrm{C}), 124.52(\mathrm{C}), 124.73(\mathrm{CH}), 127.51(\mathrm{CH}), 129.80(\mathrm{CH}), 136.78(\mathrm{C}), 140.07$ (C), 140.19 (C), 142.35 (CH), 158.61 (C); MS (EI): m/z (\%) = 282 (100, [M] $\left.]^{+}\right), 247$ (74), 232 (29), 204 (15), 171 (12), 115 (10), 75 (11); MS (ESI, +25 V): $m / z=283.2[\mathrm{M}+\mathrm{H}]^{+}$.

Crystal data: $\mathrm{C}_{17} \mathrm{H}_{15} \mathrm{ClN}_{2}$, crystal size $0.22 \times 0.20 \times 0.06 \mathrm{~mm}^{3}, M=282.76 \mathrm{~g} \mathrm{~mol}^{-1}$, monoclinic, space group: $C c, a=11.700(2), b=9.117(2), c=14.024(3) \AA, \beta=110.73(3)^{\circ}, V=1399.1(5) \AA^{3}, Z=4$, $\rho_{\text {calcd. }}=1.342 \mathrm{~g} \mathrm{~cm}^{-3}, \mu=0.264 \mathrm{~mm}^{-1}, \mathrm{~T}=198(2) \mathrm{K}, \lambda=0.71073 \AA, \theta$ range: $3.11-27.00^{\circ}, 20817$ reflections collected, 3047 independent $\left(R_{\text {int }}=0.0534\right), 187$ parameters. The structure was solved by direct methods and refined by the full-matrix least-squares method on $F^{2} ; 2296$ reflections observed, $R_{1}=0.0407, w R_{2}=0.0805[I>2 \sigma(I)]$; maximal residual electron density: 0.276 e $\AA^{-3}$. CCDC 1838728 .

N-(2-Chloro-5-methoxyphenyl)-1,5-dimethylisoquinolin-6-amine (18b). 2-Chloro-5-methoxyaniline (17b, $92 \mu \mathrm{L}$, $0.74 \mathrm{mmol}$ ) was added dropwise to a solution of 1,5-dimethylisoquinolin-6-yl trifluoromethanesulfonate (14, $0.15 \mathrm{~g}, 0.49 \mathrm{mmol})$, palladium(II) acetate $(8.3 \mathrm{mg}, 37 \mu \mathrm{mol})$, XPhos $(35 \mathrm{mg}, 74 \mu \mathrm{mol})$, and cesium carbonate $(224 \mathrm{mg}, 0.688 \mathrm{mmol})$ in toluene $(12 \mathrm{~mL})$. The mixture was heated at reflux for five hours. After cooling to room temperature, the reaction mixture was filtered over a short pad of Hyflo (ethyl acetate), and the solvent was evaporated. Purification of the residue by column chromatography (silica gel, dichloromethane/ethyl acetate, 1:1 to 0:1, each $+1 \%$ ethanol) provided $\mathrm{N}$-(2-chloro-5-methoxyphenyl)-1,5-dimethylisoquinolin-6-amine (18b, $0.141 \mathrm{~g}, 0.451 \mathrm{mmol}, \mathbf{9 2} \%)$ as a beige solid. M.p. 135-138 ${ }^{\circ} \mathrm{C}$; UV (MeOH): $\lambda=224,277,322 \mathrm{~nm}$; fluorescence $(\mathrm{MeOH}): \lambda_{\mathrm{ex}}=224$, $\lambda_{\mathrm{em}}=301$ (sh), $336 \mathrm{~nm}$; IR (ATR): $v=3416,3068,2998,2929,2853,1596,1508,1447,1421,1383,1343$, $1312,1287,1230,1207,1170,1138,1069,1027,924,820,732,671,640 \mathrm{~cm}^{-1}$; ${ }^{1} \mathrm{H}-\mathrm{NMR}\left(500 \mathrm{MHz}, \mathrm{CDCl}_{3}\right)$ : $\delta=2.53(\mathrm{~s}, 3 \mathrm{H}), 2.94(\mathrm{~s}, 3 \mathrm{H}), 3.68(\mathrm{~s}, 3 \mathrm{H}), 6.13(\mathrm{br} \mathrm{s}, 1 \mathrm{H}), 6.40(\mathrm{dd}, J=8.8,2.8 \mathrm{~Hz}, 1 \mathrm{H}), 6.47(\mathrm{~d}, J=2.8 \mathrm{~Hz}$, $1 \mathrm{H}), 7.28(\mathrm{~d}, J=8.8 \mathrm{~Hz}, 1 \mathrm{H}), 7.53(\mathrm{~d}, J=9.0 \mathrm{~Hz}, 1 \mathrm{H}), 7.63(\mathrm{~d}, J=6.1 \mathrm{~Hz}, 1 \mathrm{H}), 7.97(\mathrm{~d}, J=9.0 \mathrm{~Hz}$, $1 \mathrm{H}), 8.39(\mathrm{~d}, J=6.1 \mathrm{~Hz}, 1 \mathrm{H}) ;{ }^{13} \mathrm{C}-\mathrm{NMR}\left(125 \mathrm{MHz}, \mathrm{CDCl}_{3}\right): \delta=12.66\left(\mathrm{CH}_{3}\right), 22.61\left(\mathrm{CH}_{3}\right), 55.46\left(\mathrm{CH}_{3}\right)$, $101.82(\mathrm{CH}), 105.94(\mathrm{CH}), 113.40(\mathrm{C}), 115.53(\mathrm{CH}), 122.55(\mathrm{CH}), 123.53(\mathrm{C}), 124.67(\mathrm{C}), 124.76(\mathrm{CH}), 130.02$ (CH), $136.78(\mathrm{C}), 139.77(\mathrm{C}), 141.06(\mathrm{CH}), 142.38(\mathrm{C}), 158.64(\mathrm{C}), 159.20(\mathrm{C}) ; \mathrm{MS}(\mathrm{EI}): m / z(\%)=312$ $\left(100,[\mathrm{M}]^{+}\right), 277$ (80), 262 (76), 247 (13), 233 (18), 219 (12), 139 (10), 117 (16), 63 (10); MS (ESI, +10 V): $m / z=313.3[\mathrm{M}+\mathrm{H}]^{+}$.

N-(2-Chloro-4-methoxyphenyl)-1,5-dimethylisoquinolin-6-amine (18c). A solution of 2-chloro-4-methoxyaniline $(\mathbf{1 7 c}, 127 \mathrm{mg}, 0.806 \mathrm{mmol})$ in toluene $(4 \mathrm{~mL})$ was added dropwise to a solution of 1,5-dimethylisoquinolin-6-yl trifluoromethanesulfonate (14, $164 \mathrm{mg}, 0.537 \mathrm{mmol})$, palladium(II) acetate $(9 \mathrm{mg}, 0.04 \mathrm{mmol})$, XPhos (38 mg, $81 \mu \mathrm{mol})$, and cesium carbonate $(245 \mathrm{mg}, 0.752 \mathrm{mmol})$ in toluene $(10 \mathrm{~mL})$. The mixture was heated at reflux for one hour. After cooling to room temperature, the reaction mixture was filtered over a short pad of Hyflo (ethyl acetate), and the solvent was evaporated. Purification of the residue by column chromatography (silica gel, dichloromethane/ethyl acetate, 9:1 to 0:1, each $+1 \%$ ethanol) provided $\mathrm{N}$-(2-chloro-4-methoxyphenyl)-1,5-dimethylisoquinolin-6-amine (18c, $139 \mathrm{mg}, 0.444 \mathrm{mmol}, 83 \%)$ as a beige solid. M.p. $104-10{ }^{\circ} \mathrm{C}$; UV (MeOH): $\lambda=226,255,318 \mathrm{~nm}$; fluorescence $(\mathrm{MeOH}): \lambda_{\mathrm{ex}}=255$, $\lambda_{\text {em }}=422 \mathrm{~nm}$; IR (ATR): $v=3229,3074,2993,2948,2832,1731,1633,1606,1562,1485,1451,1436,1387$, $1341,1308,1283,1211,1182,1112,1046,936,894,864,822,789,773,689,664 \mathrm{~cm}^{-1}$; ${ }^{1} \mathrm{H}-\mathrm{NMR}(500 \mathrm{MHz}$, 
$\left.\mathrm{CDCl}_{3}\right): \delta=2.51(\mathrm{~s}, 3 \mathrm{H}), 2.93(\mathrm{~s}, 3 \mathrm{H}), 3.80(\mathrm{~s}, 3 \mathrm{H}), 5.87(\mathrm{br} \mathrm{s}, 1 \mathrm{H}), 6.79(\mathrm{dd}, J=8.7,2.8 \mathrm{~Hz}, 1 \mathrm{H}), 7.02$ $(\mathrm{d}, J=2.8 \mathrm{~Hz}, 1 \mathrm{H}), 7.09(\mathrm{~d}, J=8.9 \mathrm{~Hz}, 1 \mathrm{H}), 7.29(\mathrm{~d}, J=9.1 \mathrm{~Hz}, 1 \mathrm{H}), 7.62(\mathrm{~d}, J=6.2 \mathrm{~Hz}, 1 \mathrm{H}), 7.90(\mathrm{~d}$, $J=9.1 \mathrm{~Hz}, 1 \mathrm{H}), 8.31(\mathrm{~d}, J=6.2 \mathrm{~Hz}, 1 \mathrm{H}) ;{ }^{13} \mathrm{C}-\mathrm{NMR}\left(125 \mathrm{MHz}, \mathrm{CDCl}_{3}\right): \delta=12.01\left(\mathrm{CH}_{3}\right), 21.92\left(\mathrm{CH}_{3}\right)$, $55.84\left(\mathrm{CH}_{3}\right), 113.76(\mathrm{CH}), 115.29(\mathrm{CH}), 115.33(\mathrm{CH}), 117.69(\mathrm{C}), 118.89(\mathrm{CH}), 122.01(\mathrm{CH}), 123.18(\mathrm{C})$, $124.98(\mathrm{CH}), 126.33$ (C), 132.26 (C), 136.87 (C), 140.79 (CH, HSQC), 142.90 (C, HMBC), 155.61 (C), 158.06 (C); MS (EI): $m / z(\%)=312\left(100,[\mathrm{M}]^{+}\right), 297$ (44), 277 (12), 262 (14), 233 (17), 169 (12), 155 (11), 128 (14), 116 (15); MS (ESI, $+10 \mathrm{~V}): m / z=313.2[\mathrm{M}+\mathrm{H}]^{+}$; elemental analysis: calcd. for $\mathrm{C}_{18} \mathrm{H}_{17} \mathrm{ClN}_{2} \mathrm{O}: \mathrm{C}: 69.12$, H: 5.48, N: 8.96; found: C: 68.62, H: 5.72, N: 9.30 .

Olivacine (1). N-(2-Chlorophenyl)-1,5-dimethylisoquinolin-6-amine (18a, $20 \mathrm{mg}, 71 \mu \mathrm{mol})$, palladium(II) acetate $(4.8 \mathrm{mg}, 21 \mu \mathrm{mol})$, tri-tert-butylphosphonium tetrafluoroborate $(8.1 \mathrm{mg}, 42 \mu \mathrm{mol})$, and potassium carbonate $(39.1 \mathrm{mg}, 0.283 \mathrm{mmol})$ were dissolved in DMF $(0.5 \mathrm{~mL})$. The reaction mixture was placed in a preheated oil bath at $140{ }^{\circ} \mathrm{C}$ and stirred for $30 \mathrm{~min}$. After filtration over a short pad of Celite $\left(\mathrm{CH}_{2} \mathrm{Cl}_{2}\right)$, the halogenated solvent was evaporated, and the residue was dissolved in ethyl acetate, and washed three times with water, and then with brine. The aqueous layer was extracted with ethyl acetate, and the combined organic layers were dried (sodium sulfate). The solvent was evaporated, and the residue was purified by column chromatography (silica gel, dichloromethane/ethyl acetate, 9:1 to $0: 1$, each $+5 \%$ ethanol) to provide olivacine $(\mathbf{1}, 12.4 \mathrm{mg}, 50.3$ $\mu \mathrm{mol}, 71 \%$ ) as brown crystals. M.p. $320-324{ }^{\circ} \mathrm{C}$; $\mathrm{UV}(\mathrm{MeOH}): \lambda=223,237,275,285,292,327,342,374$, $391 \mathrm{~nm}$; fluorescence $(\mathrm{MeOH}): \lambda_{\mathrm{ex}}=285, \lambda_{\mathrm{em}}=431 \mathrm{~nm}$; IR (ATR): $v=3058,2965,2909,2765,1674$, 1597, 1479, 1467, 1407, 1334, 1311, 1280, 1252, 1222, 1196, 1150, 1108, 1064, 942, 862, 813, 765, 739, 695, $640 \mathrm{~cm}^{-1} ;{ }^{1} \mathrm{H}-\mathrm{NMR}\left(500 \mathrm{MHz}\right.$, methanol- $\left.d_{4}\right): \delta=2.85(\mathrm{~s}, 3 \mathrm{H}), 3.07\left(\mathrm{t}, J_{\mathrm{HD}}=1.1 \mathrm{~Hz}\right)$ and $3.09(\mathrm{~s}, 3 \mathrm{H})$, 7.24-7.27 (m, 1H), 7.49-7.54 (m, 2H), $7.89(\mathrm{~d}, J=6.3 \mathrm{~Hz}, 1 \mathrm{H}), 8.18(\mathrm{~d}, J=6.3 \mathrm{~Hz}, 1 \mathrm{H}), 8.27-8.29(\mathrm{~m}$, $1 \mathrm{H}), 8.87(\mathrm{~s}, 1 \mathrm{H}) ;{ }^{13} \mathrm{C}-\mathrm{NMR}\left(125 \mathrm{MHz}\right.$, methanol- $\left.d_{4}\right): \delta=12.42\left(\mathrm{CH}_{3}\right), 22.35\left(\mathrm{CH}_{3}\right), 111.86(\mathrm{CH}), 112.42$ (C), $116.05(\mathrm{CH}), 116.64(\mathrm{CH}), 120.58(\mathrm{CH}), 122.14(\mathrm{CH}), 123.57(\mathrm{C}), 124.41(\mathrm{C}), 127.25(\mathrm{C}), 128.93(\mathrm{CH})$, $134.41(\mathrm{C}), 138.84(\mathrm{CH}), 142.80(\mathrm{C}), 144.34(\mathrm{C}), 160.29$ (C); MS (EI): $m / z(\%)=246\left(100,[\mathrm{M}]^{+}\right), 229(7)$, 217 (7), 204 (9), 123 (7); MS (ESI, $+10 \mathrm{~V}$ ): $\mathrm{m} / z=247.1$ [M + H] ${ }^{+}$; HRMS (ESI): calcd. for $\mathrm{C}_{17} \mathrm{H}_{14} \mathrm{~N}_{2}$ : 246.1157, found: 246.11537; elemental analysis: calcd. for $\mathrm{C}_{17} \mathrm{H}_{14} \mathrm{~N}_{2}$ : C: 82.90, H: 5.73, N: 11.37; found: C: $83.20, \mathrm{H}: 5.81, \mathrm{~N}: 11.42$.

Crystal data: $\mathrm{C}_{17} \mathrm{H}_{14} \mathrm{~N}_{2} \cdot \mathrm{CH}_{3} \mathrm{OH}$, crystal size $0.45 \times 0.12 \times 0.07 \mathrm{~mm}^{3}, M=278.34 \mathrm{~g} \mathrm{~mol}^{-1}$, orthorhombic, space group: $P b c a, a=4.860(1), b=21.337(5), c=28.048(6) \AA, V=2908.5(11) \AA^{3}$, $\mathrm{Z}=8, \rho_{\text {calcd. }}=1.271 \mathrm{~g} \mathrm{~cm}^{-3}, \mu=0.080 \mathrm{~mm}^{-1}, T=198(2) \mathrm{K}, \lambda=0.71073 \AA, \theta$ range: $3.48-25.40^{\circ}, 60682$ reflections collected, 2656 independent $\left(R_{\text {int }}=0.0501\right), 198$ parameters. The structure was solved by direct methods and refined by the full-matrix least-squares method on $F^{2} ; 1934$ reflections observed, $R_{1}=0.0463, w R_{2}=0.1044[I>2 \sigma(I)]$; maximal residual electron density: $0.204 \mathrm{e} \AA^{-3}$. CCDC 1838729 .

4,11b-Dimethyl-11bH-pyrido[3,4-c]carbazole (20a, $2.1 \mathrm{mg}, 8.5 \mu \mathrm{mol}, 12 \%$ ), dark brown oil, less polar side product. UV (MeOH): $\lambda=250,282(\mathrm{sh}), 359 \mathrm{~nm}$; fluorescence $(\mathrm{MeOH}): \lambda_{\mathrm{ex}}=250, \lambda_{\mathrm{em}}=417 \mathrm{~nm}$; IR (ATR): $v=3348,2924,2853,2487,1630,1594,1545,1446,1200,1116,950,811,772,749,679 \mathrm{~cm}^{-1}$; ${ }^{1} \mathrm{H}-\mathrm{NMR}\left(600 \mathrm{MHz}\right.$, methanol- $\left.d_{4}\right): \delta=1.65(\mathrm{~s}, 3 \mathrm{H}), 2.76(\mathrm{~s}, 3 \mathrm{H}), 7.01(\mathrm{~d}, J=10.0 \mathrm{~Hz}, 1 \mathrm{H}), 7.49(\mathrm{t}, J=7.4 \mathrm{~Hz}$, $1 \mathrm{H}), 7.55(\mathrm{t}, J=7.5 \mathrm{~Hz}, 1 \mathrm{H}), 7.63(\mathrm{~d}, J=10.0 \mathrm{~Hz}, 1 \mathrm{H}), 7.70(\mathrm{~d}, J=7.6 \mathrm{~Hz}, 1 \mathrm{H}), 7.84(\mathrm{~d}, J=5.1 \mathrm{~Hz}, 1 \mathrm{H})$, $8.09(\mathrm{~d}, J=7.3 \mathrm{~Hz}, 1 \mathrm{H}), 8.40(\mathrm{~d}, J=5.1 \mathrm{~Hz}, 1 \mathrm{H}) ;{ }^{13} \mathrm{C}-\mathrm{NMR}\left(150 \mathrm{MHz}\right.$, methanol- $\left.d_{4}\right): \delta=21.70\left(\mathrm{CH}_{3}\right)$, $33.28\left(\mathrm{CH}_{3}\right), 59.3(\mathrm{C}, \mathrm{HMBC}), 119.63(\mathrm{CH}), 122.46(\mathrm{CH}), 123.20(\mathrm{CH}), 125.25(\mathrm{CH}), 127.3(\mathrm{C}, \mathrm{HMBC})$, $127.89(\mathrm{CH}), 129.93(\mathrm{CH}), 136.11(\mathrm{CH}), 140.89(\mathrm{C}), 149.15(\mathrm{CH}), 153.38(\mathrm{C}), 155.0(\mathrm{C}, \mathrm{HMBC}), 158.0$ (C, HMBC), 184.5 (C, HMBC); MS (EI): $m / z(\%)=246$ (100, [M] $\left.{ }^{+}\right), 231$ (24), 204 (12), 176 (7); MS (ESI, $+50 \mathrm{~V}): m / z=247.1[\mathrm{M}+\mathrm{H}]^{+}, 493.5[2 \mathrm{M}+\mathrm{H}]^{+}$.

8-Methoxyolivacine (19b). N-(2-Chloro-5-methoxyphenyl)-1,5-dimethylisoquinolin-6-amine (18b, $14 \mathbf{~ m g ,}$ $45 \mu \mathrm{mol})$, palladium(II) acetate $(3.0 \mathrm{mg}, 13 \mu \mathrm{mol})$, tri-tert-butylphosphonium tetrafluoroborate $(5.1 \mathrm{mg}$, $27 \mu \mathrm{mol})$ and potassium carbonate $(24.7 \mathrm{mg}, 0.179 \mathrm{mmol})$ were dissolved in DMF $(0.5 \mathrm{~mL})$. The reaction mixture was placed in a preheated oil bath at $140{ }^{\circ} \mathrm{C}$ and stirred for $20 \mathrm{~min}$. After filtration over a short 
pad of Celite $\left(\mathrm{CH}_{2} \mathrm{Cl}_{2}\right)$, the halogenated solvent was evaporated, and the residue was dissolved in ethyl acetate, washed three times with water, and then with brine. The aqueous layer was extracted with ethyl acetate, and the combined organic layers were dried (sodium sulfate). The solvent was evaporated and the residue was purified by column chromatography (silica gel, dichloromethane/ethyl acetate, 9:1 to 0:1, each $+5 \%$ ethanol) to provide 8 -methoxyolivacine $(\mathbf{1 9 b}, 8.0 \mathrm{mg}, 29 \mu \mathrm{mol}, 65 \%)$ as yellow crystals. M.p. 280-283 ${ }^{\circ} \mathrm{C}$; UV (MeOH): $\lambda=227,271,281,300,316,351 \mathrm{~nm}$; fluorescence $(\mathrm{MeOH}): \lambda_{\mathrm{ex}}=300, \lambda_{\mathrm{em}}=430,515 \mathrm{~nm}$; IR (ATR): $\nu=3141,3046,2993,2886,2821,2713,1622,1595,1563$, $1493,1472,1460,1412,1388,1335,1315,1297,1267,1216,1197,1160,1137,1099,1068,1030,996,942$, 916, 870, 810, $753 \mathrm{~cm}^{-1} ;{ }^{1} \mathrm{H}-\mathrm{NMR}\left(500 \mathrm{MHz}, \mathrm{DMSO}-d_{6}\right): \delta=2.79(\mathrm{~s}, 3 \mathrm{H}), 3.01(\mathrm{~s}, 3 \mathrm{H}), 3.89(\mathrm{~s}, 3 \mathrm{H}), 6.85$ $(\mathrm{dd}, J=8.6,2.2 \mathrm{~Hz}, 1 \mathrm{H}), 7.00(\mathrm{~d}, J=2.2 \mathrm{~Hz}, 1 \mathrm{H}), 7.78(\mathrm{~d}, J=6.1 \mathrm{~Hz}, 1 \mathrm{H}), 8.23(\mathrm{~d}, J=6.1 \mathrm{~Hz}, 1 \mathrm{H}), 8.24$ $(\mathrm{d}, J=8.6 \mathrm{~Hz}, 1 \mathrm{H}), 8.77(\mathrm{~s}, 1 \mathrm{H}), 11.26(\mathrm{~s}, 1 \mathrm{H}) ;{ }^{13} \mathrm{C}-\mathrm{NMR}\left(125 \mathrm{MHz}, \mathrm{DMSO}-d_{6}\right): \delta=12.36\left(\mathrm{CH}_{3}\right), 22.97$ $\left(\mathrm{CH}_{3}\right), 55.32\left(\mathrm{CH}_{3}\right), 94.84(\mathrm{CH}), 107.55(\mathrm{CH}), 110.68(\mathrm{C}), 113.55(\mathrm{CH}), 114.78(\mathrm{CH}), 116.19(\mathrm{C}), 122.00(\mathrm{C})$, $122.28(\mathrm{CH}), 125.00(\mathrm{C}), 131.74(\mathrm{C}), 139.10(\mathrm{CH}), 140.79(\mathrm{C}), 144.13(\mathrm{C}), 158.26(\mathrm{C}), 159.96$ (C); MS (EI): $m / z(\%)=276\left(100,[\mathrm{M}]^{+}\right), 261(14), 233(49), 138(8), 116(10) ; \mathrm{MS}(\mathrm{ESI},+10 \mathrm{~V}): \mathrm{m} / z=277.1[\mathrm{M}+\mathrm{H}]^{+}$; HRMS (ESI): calcd. for $\mathrm{C}_{18} \mathrm{H}_{16} \mathrm{~N}_{2} \mathrm{O}: 276.1263$, found: 276.1261 .

Crystal data: $\mathrm{C}_{18} \mathrm{H}_{16} \mathrm{~N}_{2} \mathrm{O} \cdot \mathrm{CH}_{3} \mathrm{OH}$, crystal size $0.160 \times 0.080 \times 0.060 \mathrm{~mm}^{3}, M=308.37 \mathrm{~g} \mathrm{~mol}^{-1}$, orthorhombic, space group: $P b c a, a=4.9253(3), b=21.4925(15), c=29.523(2) \AA, V=3125.3(4) \AA^{3}, Z=8$, $\rho_{\text {calcd. }}=1.311 \mathrm{~g} \mathrm{~cm}^{-3}, \mu=0.685 \mathrm{~mm}^{-1}, T=150(2) \mathrm{K}, \lambda=1.54178 \AA, \theta$ range: $2.993-68.188^{\circ}, 31382$ reflections collected, 2811 independent $\left(R_{\text {int }}=0.0544\right), 230$ parameters. The structure was solved by direct methods and refined by the full-matrix least-squares method on $F^{2} ; 2283$ reflections observed, $R_{1}=0.0387, w R_{2}=0.1001[I>2 \sigma(I)]$; maximal residual electron density: 0.221 e $\AA^{-3}$. CCDC 1838730 .

9-Methoxy-4,11b-dimethyl-11bH-pyrido[3,4-c]carbazole (20b, $1.1 \mathrm{mg}, 4.0 \mu \mathrm{mol}, 9 \%)$, brown oil, less polar side product. UV $(\mathrm{MeOH}): \lambda=221,300,325 \mathrm{~nm}$; fluorescence $(\mathrm{MeOH}): \lambda_{\mathrm{ex}}=221, \lambda_{\mathrm{em}}=296,339 \mathrm{~nm}$; IR (ATR): $v=3414,3058,2924,2855,1734,1655,1632,1593,1535,1484,1459,1437,1377,1334,1276$, $1231,1182,1149,1129,1074,935,826,740,683 \mathrm{~cm}^{-1} ;{ }^{1} \mathrm{H}-\mathrm{NMR}\left(600 \mathrm{MHz}\right.$, methanol- $\left.d_{4}\right): \delta=1.62(\mathrm{~s}, 3 \mathrm{H})$, $2.74(\mathrm{~s}, 3 \mathrm{H}), 3.94(\mathrm{~s}, 3 \mathrm{H}), 6.98(\mathrm{~d}, J=10.0 \mathrm{~Hz}, 1 \mathrm{H}), 7.04(\mathrm{dd}, J=8.2,1.7 \mathrm{~Hz}, 1 \mathrm{H}), 7.26(\mathrm{~s}, 1 \mathrm{H}), 7.61$ $(\mathrm{d}, J=10.0 \mathrm{~Hz}, 1 \mathrm{H}), 7.78(\mathrm{~d}, J=4.9 \mathrm{~Hz}, 1 \mathrm{H}), 7.94(\mathrm{~d}, J=8.2 \mathrm{~Hz}, 1 \mathrm{H}), 8.38(\mathrm{~d}, J=4.9 \mathrm{~Hz}, 1 \mathrm{H}) ;{ }^{13} \mathrm{C}-\mathrm{NMR}$ $(150 \mathrm{MHz}$, methanol-d 4$): \delta=21.67\left(\mathrm{CH}_{3}\right), 33.43\left(\mathrm{CH}_{3}\right), 56.12\left(\mathrm{CH}_{3}\right), 58.80(\mathrm{C}), 108.13(\mathrm{CH}), 113.67(\mathrm{CH})$, $119.73(\mathrm{CH}), 123.16(\mathrm{CH}), 125.46(\mathrm{CH}), 127.23(\mathrm{C}), 132.78$ (C, HMBC), $136.16(\mathrm{CH}), 149.15(\mathrm{CH}), 153.81$ (C), 156.57 (C, HMBC), 158.04 (C), 162.22 (C), 186.18 (C); MS (EI): $m / z(\%)=276\left(85,[\mathrm{M}]^{+}\right), 261(100)$, 233 (25), 218 (52), 190 (16); MS (ESI, $+50 \mathrm{~V}): m / z=277.2[\mathrm{M}+\mathrm{H}]^{+}$.

9-Methoxyolivacine (19c). N-(2-Chloro-4-methoxyphenyl)-1,5-dimethylisoquinolin-6-amine (18c, $55.0 \mathrm{mg}$, $176 \mu \mathrm{mol})$, palladium(II) acetate $(11.8 \mathrm{mg}, 53 \mu \mathrm{mol})$, tri-tert-butylphosphonium tetrafluoroborate $(20.1 \mathrm{mg}$, $106 \mu \mathrm{mol})$, and potassium carbonate $(97.2 \mathrm{mg}, 0.703 \mathrm{mmol})$ were dissolved in DMF (1.4 mL). The reaction mixture was placed in a preheated oil bath at $140{ }^{\circ} \mathrm{C}$ and stirred for $35 \mathrm{~min}$. After filtration over a short pad of Celite $\left(\mathrm{CH}_{2} \mathrm{Cl}_{2}\right)$, the halogenated solvent was evaporated, and the residue was dissolved in ethyl acetate, and then washed three times with water, and then with brine. The aqueous layer was extracted with ethyl acetate, and the combined organic layers were dried (sodium sulfate). The solvent was evaporated, and the residue was purified by column chromatography (silica gel, dichloromethane/ethyl acetate, 9:1 to $0: 1$, each $+5 \%$ ethanol) to provide 9-methoxyolivacine (19c, $30.1 \mathrm{mg}, 109 \mu \mathrm{mol}, 62 \%)$ as a yellow solid. M.p. $273-274{ }^{\circ} \mathrm{C} ; \mathrm{UV}(\mathrm{MeOH}): \lambda=224,242,272,296,332$, $394 \mathrm{~nm}$; fluorescence $(\mathrm{MeOH}): \lambda_{\mathrm{ex}}=296, \lambda_{\mathrm{em}}=471 \mathrm{~nm}$; IR (ATR): $v=3143,2914,1632,1600,1485,1436$, $1405,1380,1330,1306,1265,1206,1175,1104,1030,935,879,862,838,809,767,735,698 \mathrm{~cm}^{-1} ;{ }^{1} \mathrm{H}-\mathrm{NMR}$ $\left(500 \mathrm{MHz}, \mathrm{DMSO}-d_{6}\right): \delta=2.80(\mathrm{~s}, 3 \mathrm{H}), 3.04(\mathrm{~s}, 3 \mathrm{H}), 3.90(\mathrm{~s}, 3 \mathrm{H}), 7.14(\mathrm{dd}, J=10.0,2.5 \mathrm{~Hz}, 1 \mathrm{H}), 7.44(\mathrm{~d}$, $J=8.7 \mathrm{~Hz}, 1 \mathrm{H}), 7.79(\mathrm{~d}, J=6.1 \mathrm{~Hz}, 1 \mathrm{H}), 8.01(\mathrm{~d}, J=2.5 \mathrm{~Hz}, 1 \mathrm{H}), 8.24(\mathrm{~d}, J=6.1 \mathrm{~Hz}, 1 \mathrm{H}), 8.96(\mathrm{~s}, 1 \mathrm{H})$, $11.16(\mathrm{~s}, 1 \mathrm{H}) ;{ }^{13} \mathrm{C}-\mathrm{NMR}\left(125 \mathrm{MHz}, \mathrm{DMSO}-d_{6}\right): \delta=12.80\left(\mathrm{CH}_{3}\right), 23.45\left(\mathrm{CH}_{3}\right), 56.11\left(\mathrm{CH}_{3}\right), 104.87(\mathrm{CH})$, $111.36(\mathrm{C}), 112.00(\mathrm{CH}), 115.18(\mathrm{CH}), 115.71(\mathrm{CH}), 117.04(\mathrm{CH}), 122.00(\mathrm{C}), 123.65(\mathrm{C}), 125.36(\mathrm{C}), 132.64$ (C), $137.53(\mathrm{C}), 139.69(\mathrm{CH}), 141.61(\mathrm{C}), 153.78(\mathrm{C}), 159.21(\mathrm{C}) ; \mathrm{MS}(\mathrm{EI}): \mathrm{m} / z(\%)=276\left(100,[\mathrm{M}]^{+}\right), 261$ 
(90), 233 (27), 116 (10); MS (ESI, $+10 \mathrm{~V}): m / z=277.1[\mathrm{M}+\mathrm{H}]^{+}$; HRMS (ESI): calcd. for $\mathrm{C}_{18} \mathrm{H}_{16} \mathrm{~N}_{2} \mathrm{O}$ : 276.1263, found: 276.1269 .

10-Methoxy-4,11b-dimethyl-11bH-pyrido[3,4-c]carbazole (20c, $1.4 \mathrm{mg}, 5.0 \mu \mathrm{mol}, 3 \%)$, brown oil, less polar side product. UV (MeOH): $\lambda=260,291(\mathrm{sh}), 381 \mathrm{~nm}$; fluorescence $(\mathrm{MeOH}): \lambda_{\mathrm{ex}}=260, \lambda_{\mathrm{em}}=349$ (sh), $434 \mathrm{~nm}$; IR (ATR): $v=3389,2924,2854,1733,1655,1624,1590,1536,1466,1434,1380,1335,1295$, $1275,1240,1218,1165,1065,1030,952,865,822,744,677 \mathrm{~cm}^{-1} ;{ }^{1} \mathrm{H}-\mathrm{NMR}\left(600 \mathrm{MHz}\right.$, methanol- $\left.d_{4}\right)$ : $\delta=1.63(\mathrm{~s}, 3 \mathrm{H}), 2.74(\mathrm{~s}, 3 \mathrm{H}), 4.00(\mathrm{~s}, 3 \mathrm{H}), 6.95(\mathrm{~d}, J=10.0 \mathrm{~Hz}, 1 \mathrm{H}), 7.10(\mathrm{dd}, J=8.5,2.1 \mathrm{~Hz}, 1 \mathrm{H}), 7.55$ $(\mathrm{d}, J=10.0 \mathrm{~Hz}, 1 \mathrm{H}), 7.60(\mathrm{~d}, J=8.5 \mathrm{~Hz}, 1 \mathrm{H}), 7.66(\mathrm{~d}, J=2.1 \mathrm{~Hz}, 1 \mathrm{H}), 7.83(\mathrm{~d}, J=5.1 \mathrm{~Hz}, 1 \mathrm{H}), 8.38(\mathrm{~d}$, $J=5.1 \mathrm{~Hz}, 1 \mathrm{H}) ;{ }^{13} \mathrm{C}-\mathrm{NMR}\left(150 \mathrm{MHz}\right.$, methanol- $\left.d_{4}\right): \delta=21.69\left(\mathrm{CH}_{3}\right), 33.33\left(\mathrm{CH}_{3}\right), 56.44\left(\mathrm{CH}_{3}\right), 59.25(\mathrm{C})$, $112.13(\mathrm{CH}), 114.60(\mathrm{CH}), 119.52(\mathrm{CH}), 122.93(\mathrm{CH}), 123.26(\mathrm{CH}), 127.5(\mathrm{C}, \mathrm{HMBC}), 134.87(\mathrm{CH}), 142.7$ (C, HMBC), 147.3 (C, HMBC), 148.89 (CH), 153.2 (C, HMBC), 157.8 (C, HMBC), 160.73 (C), 182.4 (C, HMBC); MS (EI): $m / z(\%)=276\left(100,[\mathrm{M}]^{+}\right), 261$ (42), 246 (24), 233 (46), 218 (31), 190 (13); MS (ESI, +50 $\mathrm{V}): m / z=277.2[\mathrm{M}+\mathrm{H}]^{+}$.

8-Hydroxyolivacine (4). 8-Methoxyolivacine (19b, $17.0 \mathrm{mg}, 61.5 \mu \mathrm{mol}$ ) was dissolved in $48 \%$ aqueous $\mathrm{HBr}$ $(1.1 \mathrm{~mL})$, and the mixture was heated at reflux for $24 \mathrm{~h}$. After cooling to room temperature, the mixture was carefully neutralized using a $25 \%$ aqueous solution of ammonia. The mixture was extracted with ethyl acetate until the aqueous layer was completely colorless. Evaporation of the organic solvent led to a yellow solid, which was purified by chromatography (Alox $\mathrm{N}, 5 \% \mathrm{H}_{2} \mathrm{O}, \mathrm{CH}_{2} \mathrm{Cl}_{2}$ /methanol, 1:1) to provide 8-hydroxyolivacine $(4,13.5 \mathrm{mg}, 51.5 \mu \mathrm{mol}, 84 \%)$ as a yellow solid. An additional purification by preparative HPLC provided very pure $4(8.5 \mathrm{mg}, 32 \mu \mathrm{mol})$ for biological testing. M.p. $239^{\circ} \mathrm{C}$; UV $(\mathrm{MeOH}): \lambda=239,301,317 \mathrm{~nm}$; fluorescence $(\mathrm{MeOH}): \lambda_{\mathrm{ex}}=301, \lambda_{\mathrm{em}}=434,520 \mathrm{~nm}$; IR (ATR): $v=3505$, 3279, 3198, 2827, 1660, 1619, 1474, 1433, 1407, 1341, 1190, 1166, 1138, 1102, 840, 800, 722, $633 \mathrm{~cm}^{-1}$; ${ }^{1} \mathrm{H}-\mathrm{NMR}\left(500 \mathrm{MHz}\right.$, methanol- $\left.d_{4}\right): \delta=2.94(\mathrm{~s}, 3 \mathrm{H}), 3.34(\mathrm{~s}, 3 \mathrm{H}), 6.90(\mathrm{dd}, J=8.5,2.1 \mathrm{~Hz}, 1 \mathrm{H}), 7.01$ $(\mathrm{d}, J=2.1 \mathrm{~Hz}, 1 \mathrm{H}), 8.18(\mathrm{~d}, J=7.0 \mathrm{~Hz}, 1 \mathrm{H}), 8.20(\mathrm{~d}, J=8.5 \mathrm{~Hz}, 1 \mathrm{H}), 8.37(\mathrm{~d}, J=7.0 \mathrm{~Hz}, 1 \mathrm{H}), 9.00(\mathrm{~s}, 1 \mathrm{H})$; ${ }^{13} \mathrm{C}-\mathrm{NMR}\left(125 \mathrm{MHz}\right.$, methanol- $\left.d_{4}\right): \delta=12.41\left(\mathrm{CH}_{3}\right), 18.63\left(\mathrm{CH}_{3}\right), 98.31(\mathrm{CH}), 111.38(\mathrm{CH}), 113.55(\mathrm{C})$, $115.93(\mathrm{C}), 116.93(\mathrm{CH}), 119.58(\mathrm{CH}), 121.73(\mathrm{C}), 123.95(\mathrm{CH}), 127.12(\mathrm{CH}), 130.23(\mathrm{C}), 134.44(\mathrm{C}), 146.42$ (2C), 157.30 (C), 161.11 (C); MS (EI): $m / z(\%)=262\left(100,[\mathrm{M}]^{+}\right), 180$ (10); MS (ESI, +10 V): $m / z=263.1$ $[\mathrm{M}+\mathrm{H}]^{+}, 547$ [2M + Na] $]^{+}$; HRMS (ESI): calcd. for $\mathrm{C}_{17} \mathrm{H}_{14} \mathrm{~N}_{2} \mathrm{O}: 262.1106$, found: 262.1104 .

9-Hydroxyolivacine (5). 9-Methoxyolivacine (19c, $38.0 \mathrm{mg}, 138 \mu \mathrm{mol}$ ) was dissolved in $48 \%$ aqueous $\mathrm{HBr}(2.3 \mathrm{~mL})$, and the mixture was heated at reflux for $24 \mathrm{~h}$. After cooling to room temperature, the mixture was carefully neutralized using a $25 \%$ aqueous solution of ammonia. The mixture was extracted with ethyl acetate until the aqueous layer was colorless. The combined organic layers were washed with water and brine, and then dried (sodium sulfate), and the solvent was evaporated. The residue was purified by column chromatography (silica gel, $\mathrm{CH}_{2} \mathrm{Cl}_{2}$ /THF, 4:1 to 2:3) to provide 9-hydroxyolivacine $(5,25.2 \mathrm{mg}, 96.1 \mu \mathrm{mol}, 70 \%)$ as a yellow solid. An additional purification by preparative HPLC provided very pure $5(6.1 \mathrm{mg}, 23 \mu \mathrm{mol})$ for biological testing. M.p. $249^{\circ} \mathrm{C}$; UV (MeOH): $\lambda=245,274,311,355,375 \mathrm{~nm}$; fluorescence $(\mathrm{MeOH}): \lambda_{\mathrm{ex}}=311, \lambda_{\mathrm{em}}=482 \mathrm{~nm}$; IR (ATR): $v=3220,2921,2853,1734,1666,1611,1425,1328,1288,1185,1127,975,840,799,721 \mathrm{~cm}^{-1},{ }^{1} \mathrm{H}-\mathrm{NMR}$ $\left(500 \mathrm{MHz}\right.$, methanol- $\left.d_{4}\right): \delta=2.96(\mathrm{~s}, 3 \mathrm{H}), 3.36(\mathrm{~s}, 3 \mathrm{H}, \mathrm{HSQC}), 7.22(\mathrm{dd}, J=8.6,2.3 \mathrm{~Hz}, 1 \mathrm{H}), 7.51$ $(\mathrm{d}, J=8.6 \mathrm{~Hz}, 1 \mathrm{H}), 7.82(\mathrm{~d}, J=2.3 \mathrm{~Hz}, 1 \mathrm{H}), 8.19(\mathrm{~d}, J=7.1 \mathrm{~Hz}, 1 \mathrm{H}), 8.38(\mathrm{~d}, J=7.1 \mathrm{~Hz}, 1 \mathrm{H}), 9.17(\mathrm{~s}, 1 \mathrm{H})$; ${ }^{13} \mathrm{C}-\mathrm{NMR}\left(125 \mathrm{MHz}\right.$, methanol- $\left.d_{4}\right): \delta=12.42\left(\mathrm{CH}_{3}\right), 18.67\left(\mathrm{CH}_{3}\right), 107.97(\mathrm{CH}), 113.13(\mathrm{CH}), 113.96$ (C), $119.43(\mathrm{CH}), 119.48(\mathrm{CH}), 119.54(\mathrm{CH}), 121.00(\mathrm{C}), 124.35(\mathrm{C}), 127.40(\mathrm{CH}), 129.67(\mathrm{C}), 134.68(\mathrm{C})$, 138.24 (C), 146.56 (C), 153.52 (C), 158.18 (C); MS (EI): $m / z(\%)=262$ (100, [M] $\left.{ }^{+}\right), 131$ (12); MS (ESI, $+10 \mathrm{~V}): m / z=263.1[\mathrm{M}+\mathrm{H}]^{+}$; HRMS (ESI): calcd. for $\mathrm{C}_{17} \mathrm{H}_{14} \mathrm{~N}_{2} \mathrm{O}: 262.1106$, found: 262.1107.

\section{Conclusions}

In conclusion, we have developed a straightforward synthesis of olivacine (1) and four of its oxygenated pyrido[4,3-b]carbazole derivatives via Buchwald-Hartwig coupling of the isoquinolinyl triflate 14 and the ortho-chloroarylamine 18a-c followed by a Heck-type cyclization. In a test for 
the inhibition of the growth of $M$. tuberculosis (strain $\mathrm{H}_{37} \mathrm{Rv}$ ), 9-methoxyolivacine (19c) proved to be the most active compound, with an $\mathrm{MIC}_{90}$ value of $1.5 \mu \mathrm{M}$ and a relatively low toxicity for a mammalian cell line. These initial results indicate that the pyrido[4,3-b]carbazoles are a promising class of compounds for our ongoing search for a carbazole-based tuberculosis drug candidate.

Supplementary Materials: The following data are available online. Copies of the ${ }^{1} \mathrm{H}-\mathrm{NMR},{ }^{13} \mathrm{C}-\mathrm{NMR}$ and $2 \mathrm{D}$ NMR spectra.

Author Contributions: U.S. and H.-J.K. conceived and designed the experiments; U.S. and G.T. performed the chemical synthesis and characterized the compounds; A.J. and O.K. performed the X-ray analyses; B.W. and S.G.F. designed and performed the study for inhibition of M. tuberculosis; U.S. and H.-J.K. wrote the paper.

Funding: This research received no external funding.

Acknowledgments: We are grateful to Thomas Hopfmann and Erik Troschke for their experimental contributions.

Conflicts of Interest: The authors declare no conflict of interest.

\section{References}

1. Schmutz, J.; Hunziker, F. Die Alkaloide von Aspidosperma olivaceum M. Arg. Aspidosperma-Alkaloide, 3. Mitteilung. Pharm. Acta Helv. 1958, 33, 341-347.

2. Wittwer, H.; Schmutz, J. Die Synthese von Olivacin, Dihydro-olivacin, Tetrahydro-olivacin, N-Methyltetrahydro-olivacin, und die Konstitution von u-Alkaloid D. Helv. Chim. Acta 1960, 43, 793-799.

3. Maftouh, M.; Besselievre, R.; Monsarrat, B.; Lesca, P.; Meunier, B.; Husson, H.P.; Paoletti, C. Synthesis and cytotoxic activity of hydroxylated derivatives of olivacine in relation with their biotransformation. J. Med. Chem. 1985, 28, 708-714. [CrossRef] [PubMed]

4. Stiborová, M.; Sejbal, J.; Bořek-Dohalská, L.; Aimová, D.; Poljaková, J.; Forsterová, K.; Rupertová, M.; Wiesner, J.; Hudeček, J.; Wiessler, M.; et al. The anticancer drug ellipticine forms covalent DNA adducts, mediated by human cytochromes $\mathrm{P} 450$, through metabolism to 13-hydroxyellipticine and ellipticine $N^{2}$-oxide. Cancer Res. 2004, 64, 8374-8380. [CrossRef] [PubMed]

5. Rocha e Silva, L.F.; Montoia, A.; Amorim, R.C.N.; Melo, M.R.; Henrique, M.C.; Nunomura, S.M.; Costa, M.R.F.; Andrade Neto, V.F.; Costa, D.S.; Dantas, G.; et al. Comparative in vitro and in vivo antimalarial activity of the indole alkaloids ellipticine, olivacine, cryptolepine and a synthetic cryptolepine analog. Phytomedicine 2012, 20, 71-76. [CrossRef] [PubMed]

6. Deane, F.M.; O'Sullivan, E.C.; Maguire, A.R.; Gilbert, J.; Sakoff, J.A.; McCluskey, A.; McCarthy, F.O. Synthesis and evaluation of novel ellipticines as potential anti-cancer agents. Org. Biomol. Chem. 2013, 11, 1334-1344. [CrossRef] [PubMed]

7. Montoia, A.; Rocha e Silva, L.F.; Torres, Z.E.; Costa, D.S.; Henrique, M.C.; Lima, E.S.; Vasconcellos, M.C.; Souza, R.C.Z.; Costa, M.R.F.; Grafov, A.; et al. Antiplasmodial activity of synthetic ellipticine derivatives and an isolated analog. Bioorg. Med. Chem. Lett. 2014, 24, 2631-2634. [CrossRef] [PubMed]

8. Miller, C.M.; McCarthy, F.O. Isolation, biological activity and synthesis of the natural product ellipticine and related pyridocarbazoles. RSC Adv. 2012, 2, 8883-8918. [CrossRef]

9. Rouëssé, J.; Spielmann, M.; Turpin, F.; Le Chevalier, T.; Azab, M.; Mondésir, J.M. Phase II study of elliptinium acetate salvage treatment of advanced breast cancer. Eur. J. Cancer 1993, 29, 856-859. [CrossRef]

10. Gribble, G.W. Approaches to the synthesis of the antitumor pyridocarbazole alkaloids. Synlett 1991, 289-300. [CrossRef]

11. Schmidt, A.W.; Reddy, K.R.; Knölker, H.-J. Occurrence, biogenesis, and synthesis of biologically active carbazole alkaloids. Chem. Rev. 2012, 112, 3193-3328. [CrossRef] [PubMed]

12. Bergman, J.; Carlsson, R. Conversion of diindolyl methanes to 3-vinylindoles. A simple synthesis of the indole alkaloid olivacine. Tetrahedron Lett. 1978, 4055-4058. [CrossRef]

13. Besselièvre, R.; Husson, H.-P. Syntheses in the ellipticine-olivacine series, a possible biogenetic model. Tetrahedron 1981, 37 (Suppl. 1), 241-246. [CrossRef]

14. Kutney, J.P.; Noda, M.; Lewis, N.G.; Monteiro, B.; Mostowicz, D.; Worth, B.R. Dihydropyridines in synthesis and biosynthesis. V. Synthesis of pyridocarbazole alkaloids: olivacine and ( \pm )-guatambuine. Can. J. Chem. 1982, 60, 2426-2430. [CrossRef] 
15. Miki, Y.; Tsuzaki, Y.; Hibino, H.; Aoki, Y. Synthesis of 3-methoxyolivacine and olivacine by Friedel-Crafts reaction of indole-2,3-dicarboxylic anhydride with 2,4,6-trimethoxypyridine. Synlett 2004, 12, 2206-2208. [CrossRef]

16. Bennasar, M.L.; Roca, T.; Ferrando, F. Regioselective 6-endo cyclizations of 2-indolylacyl radicals: total synthesis of the pyrido[4,3-b]carbazole alkaloid guatambuine. J. Org. Chem. 2006, 71, 1746-1749. [CrossRef] [PubMed]

17. Ramkumar, N.; Nagarajan, R. Total synthesis of ellipticine quinones, olivacine, and calothrixin B. J. Org. Chem. 2013, 79, 736-741. [CrossRef] [PubMed]

18. Itoh, T.; Abe, T.; Choshi, T.; Nishiyama, T.; Yanada, R.; Ishikura, M. Concise total syntheses of pyrido[4,3-b]carbazole alkaloids using copper-mediated 6r-electrocyclization. Eur. J. Org. Chem. 2016, 13, 2290-2299. [CrossRef]

19. Pierré, A.; Atassi, G.; Devissaguet, M.; Bisagni, E. Novel olivacine and ellipticine derivatives: S-16020-2 and related compounds as potential antitumor agents. Drugs Future 1997, 22, 53-59. [CrossRef]

20. Jasztold-Howorko, R.; Croisy, A.; Carrez, D. An alternative way of the synthesis of 1-substituted 9-methoxy-5-methyl-6H-pyrido[4,3-b]carbazole derivatives. Acta Pol. Pharm. 2005, 62, 207-212. [PubMed]

21. Brütting, C.; Schmidt, A.W.; Kataeva, O.; Knölker, H.-J. First total synthesis of 7-isovaleryloxy-8methoxygirinimbine. Synthesis 2018, 50. [CrossRef]

22. Comins, D.L.; Brown, J.D. Ortho substitution of $m$-anisaldehyde via $\alpha$-amino alkoxide directed lithiation. J. Org. Chem. 1989, 54, 3730-3732. [CrossRef]

23. Bur, D.; Grisostomi, C.; Kimmerlin, T.; Remen, L.; Siendt, H.; Vercauteren, M.; Welford, R. Tricyclic piperidine compounds. Patent WO2016177690A1, 10 November 2016.

24. Becknell, N.C.; Dandu, R.R.; Dorsey, B.D.; Gotchev, D.B.; Hudkins, R.L.; Weinberg, L.; Zificsak, C.A.; Zulli, A.L. 1,4-Substituted piperidine derivatives. Patent WO2016205633A1, 22 December 2016.

25. Ruiz-Castillo, P.; Buchwald, S.L. Applications of palladium-catalyzed C-N cross-coupling reactions. Chem. Rev. 2016, 116, 12564-12649. [CrossRef] [PubMed]

26. Miller, R.B.; Moock, T. A general synthesis of 6-H-pyrido[4,3-b]carbazole alkaloids. Tetrahedron Lett. 1980, 21, 3319-3322. [CrossRef]

27. Åkermark, B.; Eberson, L.; Jonsson, E.; Pettersson, E. Palladium-promoted cyclization of diphenyl ether, diphenylamine, and related compounds. J. Org. Chem. 1975, 40, 1365-1367. [CrossRef]

28. Krahl, M.P.; Jäger, A.; Krause, T.; Knölker, H.-J. First total syntheis of the 7-oxygenated carbazole alkaloids clauszoline-K, 3-formyl-7-hydroxycarbazole, clausine $\mathrm{M}$, clausine $\mathrm{N}$ and the anti-HIV active siamenol using a highly efficient palladium-catalyzed approach. Org. Biomol. Chem. 2006, 4, 3215-3219. [CrossRef] [PubMed]

29. Liégault, B.; Lee, D.; Huestis, M.P.; Stuart, D.R.; Fagnou, K. Intramolecular Pd(II)-catalyzed oxidative biaryl synthesis under air: reaction development and scope. J. Org. Chem. 2008, 73, 5022-5028. [CrossRef] [PubMed]

30. Iwaki, T.; Yasuhara, A.; Sakamoto, T. Novel synthetic strategy of carbolines via palladium-catalyzed amination and arylation reaction. J. Chem. Soc. Perkin Trans. 1 1999, 11, 1505-1510. [CrossRef]

31. Campeau, L.C.; Parisien, M.; Jean, A.; Fagnou, K. Catalytic direct arylation with aryl chlorides, bromides, and iodides: intramolecular studies leading to new intermolecular reactions. J. Am. Chem. Soc. 2006, 128, 581-590. [CrossRef] [PubMed]

32. Queiroz, M.-J.R.P.; Ferreira, I.C.F.R.; Gaetano, Y.D.; Kirsch, G.; Calhelha, R.C.; Estevinho, L.M. Synthesis and antimicrobial activity studies of ortho-chlorodiarylamines and heteroaromatic tetracyclic systems in the benzo[b]thiophene series. Bioorg. Med. Chem. 2006, 14, 6827-6831. [CrossRef] [PubMed]

33. Burnell, R.H.; Della Casa, D. Alkaloids of Aspidosperma vargasii A. DC. Can. J. Chem. 1967, 45, 89-92. [CrossRef]

34. Sunthitikawinsakul, A.; Kongkathip, N.; Kongkathip, B.; Phonnakhu, S.; Daly, J.W.; Spande, T.F.; Nimit, Y.; Rochanaruangrai, S. Coumarins and carbazoles from Clausena excavata exhibited antimycobacterial and antifungal activities. Planta Med. 2003, 69, 155-157. [CrossRef] [PubMed]

35. Okunade, A.L.; Elvin-Lewis, M.P.F.; Lewis, W.H. Natural antimycobacterial metabolites: current status. Phytochemistry 2004, 65, 1017-1032. [CrossRef] [PubMed]

36. Ma, C.; Case, R.J.; Wang, Y.; Zhang, H.-J.; Tan, G.T.; Hung, N.V.; Cuong, N.M.; Franzblau, S.G.; Soejarto, D.D.; Fong, H.H.S.; et al. Anti-tuberculosis constituents from the stem bark of Micromelum hirsutum. Planta Med. 2005, 71, 261-267. [CrossRef] [PubMed] 
37. Choi, T.A.; Czerwonka, R.; Fröhner, W.; Krahl, M.P.; Reddy, K.R.; Franzblau, S.G.; Knölker, H.-J. Synthesis and activity of carbazole derivatives against Mycobacterium tuberculosis. Chem. Med. Chem. 2006, 1, 812-815. [CrossRef] [PubMed]

38. Choi, T.A.; Czerwonka, R.; Forke, R.; Jäger, A.; Knöll, J.; Krahl, M.P.; Krause, T.; Reddy, K.R.; Franzblau, S.G.; Knölker, H.-J. Synthesis and pharmacological potential of carbazoles. Med. Chem. Res. 2008, 17, 374-385. [CrossRef]

39. Börger, C.; Brütting, C.; Julich-Gruner, K.K.; Hesse, R.; Kumar, V.P.; Kutz, S.K.; Rönnefahrt, M.; Thomas, C.; Wan, B.; Franzblau, S.G.; Knölker, H.-J. Anti-tuberculosis activity and structure-activity relationships of oxygenated tricyclic carbazole alkaloids and synthetic derivatives. Bioorg. Med. Chem. 2017, 25, 6167-6174. [CrossRef] [PubMed]

40. Pauli, G.F.; Case, R.J.; Inui, T.; Wang, Y.; Cho, S.; Fischer, N.H.; Franzblau, S.G. New perspectives on natural products in TB drug research. Life Sci. 2005, 78, 485-494. [CrossRef] [PubMed]

41. Cho, S.; Lee, H.S.; Franzblau, S. Microplate alamar blue assay (MABA) and low oxygen recovery assay (LORA) for Mycobacterium tuberculosis. In Mycobacteria Protocols; Parish, T., Roberts, D.M., Eds.; Springer: New York, NY, USA, 2015; pp. 281-292.

42. Falzari, K.; Zhu, Z.; Pan, D.; Liu, H.; Hongmanee, P.; Franzblau, S.G. In vitro and in vivo activities of macrolide derivatives against Mycobacterium tuberculosis. Antimicrob. Agents Chemother. 2005, 49, 1447-1454. [CrossRef] [PubMed]

43. Sheldrick, G.M. SHELXS-97, Programs for Crystal Structure Solution; University of Göttingen: Göttingen, Germany, 1997.

44. Sheldrick, G.M. SADABS, v. 2.10, Bruker/Siemens Area Detector Absorption Correction Program; Bruker AXS Inc.: Madison, WI, USA, 2002.

45. Sheldrick, G.M. SHELXL-97, Programs for Crystal Structure Refinement; University of Göttingen: Göttingen Germany, 1997.

46. Farrugia, L.J. ORTEP-3 for Windows-A version of Ortep-III with a graphical user interface (GUI). J. Appl. Crystallogr. 1997, 30, 565. [CrossRef]

Sample Availability: Not available.

(c) 2018 by the authors. Licensee MDPI, Basel, Switzerland. This article is an open access article distributed under the terms and conditions of the Creative Commons Attribution (CC BY) license (http://creativecommons.org/licenses/by/4.0/). 